Review

\title{
Structural Health Monitoring by Using Fiber-Optic Distributed Strain Sensors With High Spatial Resolution
}

\author{
Hideaki MURAYAMA $^{1^{*}}$, Daichi WADA ${ }^{1}$, and Hirotaka IGAWA ${ }^{2}$ \\ ${ }^{1}$ School of Engineering, The University of Tokyo, 7-3-1 Hongo, Bunkyo-ku, Tokyo, 113-8656 Japan \\ ${ }^{2}$ Japan Aerospace Exploration Agency, 6-13-1 Ohsawa, Mitaka, Tokyo, 181-0015 Japan \\ *Corresponding author: Hideaki MURAYAMAＥ-mail: murayama@sys.t.u-tokyo.ac.jp
}

\begin{abstract}
In this paper, we review our researches on the topics of the structural health monitoring (SHM) with the fiber-optic distributed strain sensor. Highly-dense information on strains in a structure can be useful to identify some kind of existing damages or applied loads in implementation of SHM. The fiber-optic distributed sensors developed by the authors have been applied to the damage detection of a single-lap joint and load identification of a beam simply supported. We confirmed that the applicability of the distributed sensor to SHM could be improved as making the spatial resolution higher. In addition, we showed that the simulation technique considering both structural and optical effects seamlessly in strain measurement could be powerful tools to evaluate the performance of a sensing system and design it for SHM. Finally, the technique for simultaneous distributed strain and temperature measurement using the PANDA-fiber Bragg grating (FBG) is shown in this paper, because problems caused by the cross-sensitivity toward strain and temperature would be always inevitable in strain measurement for SHM.
\end{abstract}

Keywords: Structural health monitoring, fiber-optic distributed sensor, strain monitoring, damage detection, load identification

Citation: Hideaki MURAYAMA, Daichi WADA, and Hirotaka IGAWA, "Structural Health Monitoring by Using Fiber-Optic Distributed Strain Sensors with High Spatial Resolution,” Photonic Sensors, DOI: 10.1007/s13320-013-0140-5.

\section{Introduction}

The purpose of structural health monitoring (SHM) is to lead a structure, such as the aerospace, ship, and civil and mechanical engineering infrastructure, to be safer at the lower cost. SHM systems capable of assessing structural integrity during manufacturing or in-service operation would allow actions that can result in the improved quality, optimal operation, timely maintenance, and longer service life [1].

The function required in the SHM system during the in-service operation is to identify the load applied to a structure or damage in it. If the structural capacity, namely, the strength or the stiffness, is given, we can determine whether the identified load is within the range tolerated by the structure. On the other hand, when it is believed that the applied load is controlled under the design load case, we can estimate whether the lack of the inherent strength or stiffness derived by the identified damage/deterioration causes critical failure or harmful deformation. Considering the excessive loading or existing defect, it would be obviously better to simultaneously identify both load and damage at the current condition. In addition, the

Received: 18 August 2013 / Revised version: 5 September 2013

(C) The Author(s) 2013. This article is published with open access at Springerlink.com 
ability of the SHM system can be enhanced by functions to take into account environmental factors and changes over time. Then, it is usually expected that these functions are accomplished based on real-time information obtained from sensors with which the structure is equipped.

In most cases, an actual applied load cannot be directly measured by sensors. Generally, it can be estimated by using deformation measured and based on the theory of elasticity [2].

It makes even less sense that sensor outputs derive existing damage in a straightforward manner [3]. Although there would be a non-destructive inspection (NDI) technique which finds damage almost directly, it cannot be used for real-time monitoring or global damage detection of in-service structures [4]. Therefore, it is important to choose a measurand whose variation can be related to damage interested.

Strain is a parameter which directly relates deformation and has sensitivity to damage of structural members subjected to a load. Fortunately, it is also an elemental measurand for typical fiber-optic distributed sensors as well as temperature [5]. Fiber-optic distributed sensors return a value of the measurand as a function of the linear position along an optical fiber, as they are having useful characteristics of optical fiber sensors for SHM, such as immunity of electromagnetic interference, durability and capability to be embedded into composite materials [6]. Among the fiber-optic distributed sensors, various distributed sensing techniques based on Brillouin scattering have been proposed for strain measurement [7]. Fiber Bragg grating (FBG) sensors with advanced interrogation systems can be also applied to distributed sensing as well as quasi-distributed one [8-12].

In 1999, the author applied the distributed sensing system with the Brillouin optical time domain reflectometry (BOTDR) to SHM based on strain monitoring for 24-m yacht structures [13]. To the author's knowledge, this is the first work in which distributed strain monitoring in practical composite structures was implemented by fiberoptic sensors and provided the evidence of their effectiveness for SHM. On the other hand, it also gave us problems to be addressed. The most important one was the lack of the spatial resolution [14]. So the authors have developed the sensing system to measure strain distribution with a high spatial resolution for a decade [15]. We have also applied the developed fiber-optic distributed sensor to load identification and damage detection in the field of SHM, especially for structures made of advanced composite materials [16-18]. In addition, fundamental studies have been also implemented in order to improve the applicability of them to SHM based on strain monitoring $[19,20]$.

In this paper, we review our researches on these topics. In Section 2, the application of distributed strain monitoring with BOTDR to a large-scale composite structure is described. Then, the principle of the developed fiber-optic distributed sensor is shown in Section 3. Then, in Sections 4 and 5, the techniques for damage detection and load identification based on distributed strain monitoring in structures are shown, respectively. The studies on the simulation based design process and simultaneous strain and temperature measurement are described in Section 6. Finally, we summarize this paper and show future directions in Section 7.

\section{Distributed strain monitoring by fiber- optic sensor}

In this section, distributed strain monitoring of yacht structures in 1999 is described to show the applicability of fiber-optic distributed sensors to SHM. It is also shown that the spatial resolution is important for the ability to detect damage in SHM with the distributed sensor.

\subsection{SHM for IACC yacht}

The author applied fiber-optic sensors to International America's Cup Class (IACC) yachts made in Japan, namely, Asura and Idaten [13]. These 
yachts were equipped with fiber-optic distributed sensors using Brillouin scattering. Then, longitudinal and transverse strains of the yachts were periodically measured to assess stiffness of the structures in America's Cup 2000 which was held at Auckland in New Zealand.

The IACC yacht was about $24 \mathrm{~m}$ long with a mast about $35 \mathrm{~m}$ high above the water surface. The schematic of the IACC yacht is shown in Fig. 1. During sailing, the yacht was subjected to large static forces by wires (called stay or shroud) and the longitudinal bending moment peaks at the position of the mast as shown in Fig. 1. These static forces exert more dominant influences upon the structure than dynamic forces (wind and wave). The longitudinal stiffness and transverse stiffness as if withstanding large longitudinal and transverse bending loads, respectively, are critical for the IACC yacht's structure. Therefore, carbon fiber reinforced plastics (CFRP) sandwich composite materials were used in major structural parts because of their beneficial properties to flexural loading.

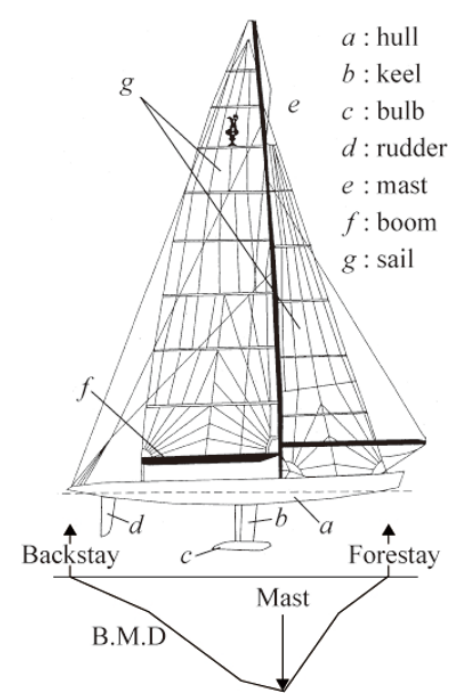

Fig. 1 Schematic of the IACC yacht.

In America's Cup 2000, the author equipped Asura and Idaten with longitudinal and transverse sensing fibers. As shown in Fig. 2, the former $(a-h)$ was fixed onto the inside of the hull with the adhesive, and it traveled back and forth in the longitudinal direction. Two lines $(a-b$ and $g-h)$ were located above the neutral axis on which the stresses were zero in longitudinal bending. Another two lines ( $c-d$ and $e-f$ ) were located below the axis. On the other hand, the latter $(j-m)$ was traveled back and forth along the boundary between the hull and the bulkhead supporting the mast. A line $(j-k)$ and another $(l-m)$ were fixed onto the hull surface and the flange of the bulkhead, respectively. These sensing fibers were installed during construction.

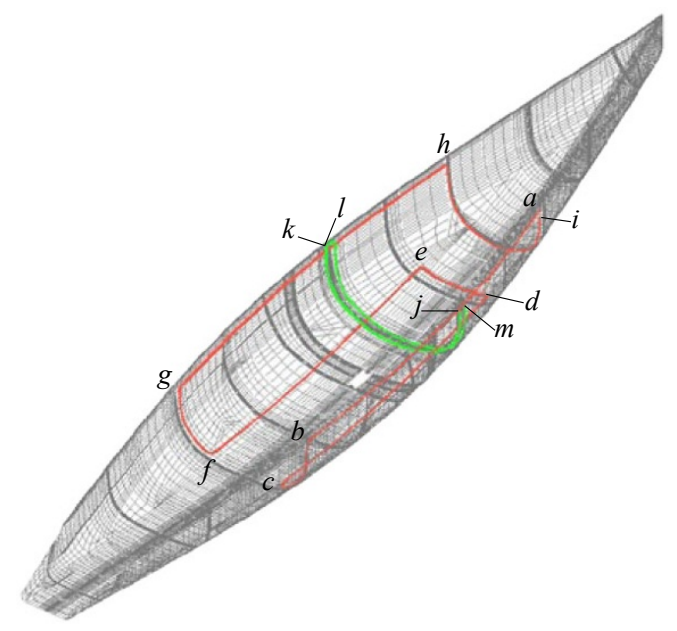

Fig. 2 Location of the sensing fibers in the yacht's structure.

In order to access the longitudinal stiffness of the manufactured yachts, strain distributions along the longitudinal sensing fiber were measured using the BOTDR just after the completion of construction. Figure 3 shows the difference between the strain distributions under the forestay tension of 4 tons and 0 tons in Idaten. The result calculated by finite element analysis (FEA) is also shown for comparison. The alphabets inscribed in the figure are corresponding to those in Fig. 2. Since the yacht was subjected to a bending moment by the forestay and backstay, the regions below the neutral axis, $c-d$ and $e-f$, had tensile strain, and those above the axis, $a-b$ and $g-h$, had compressive strain. The good agreement between the measured results and the calculated ones showed that the manufactured yacht fulfilled the designer's requirements in terms of longitudinal stiffness. 


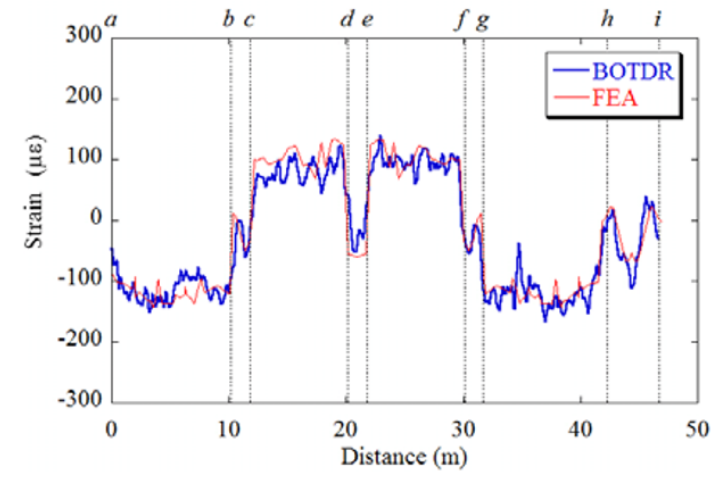

Fig. 3 Strain distributions along the longitudinal sensing fiber.

Strain distributions along the transverse sensing fiber were also measured to access the transverse stiffness of the adhesive joint and to detect unusual deformation between the hull and the bulkhead supporting the mast. Figure 4 shows the measured and numerical results of Idaten. These results were obtained by deducting the strain data of the yacht without its mast from that of the yacht with its mast. Larger strains could be seen under the mast, since it was pushing the bottom. There was no remarkable deviation in strains between the hull surface $(j-k)$ and the flange of the bulkhead $(l-m)$. Therefore, we can say that the stiffness of the adhesive joint was sufficient and that there might not be any damage in the adhered boundary.

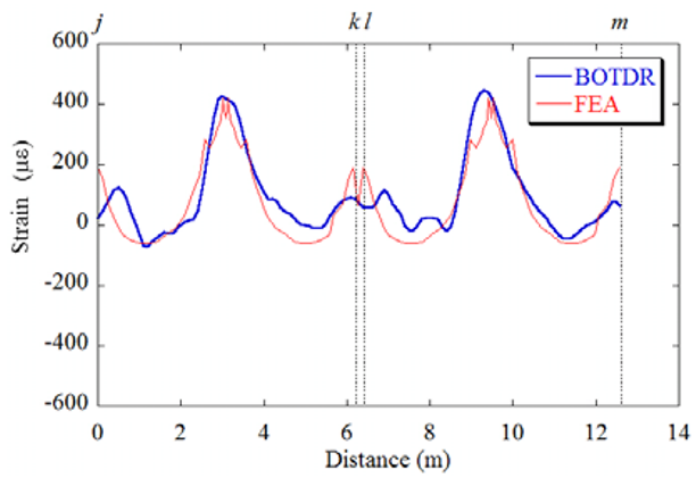

Fig. 4 Strain distributions along the transverse sensing fiber.

The periodic inspection in which the strain distributions of the longitudinal and transverse sensing fibers were monitored was repeatedly carried on for about eight months, and we did not find an abnormal strain. By the sensing system with the BOTDR, the assessment of structural integrity in terms of stiffness based on the measured strain distributions was easily and repeatedly implemented as SHM for the IACC yacht.

\subsection{Spatial resolution}

At that time, the spatial resolution of the BOTDR was $1.0 \mathrm{~m}$, and the accuracy for the strain measurement was about $\pm 30 \mu \varepsilon$. It seems that the actual strain distributions of the applied structure were not overly complicated, so the sensing system could provide useful information.

In order to investigate the ability to detect damage, interfacial debonding between the hull and the bulkhead was introduced in to the finite element (FE) model [14]. The debonding was $500 \mathrm{~mm}$ in length along the transvers sensing fiber around the mast. Strain distributions measured by the BOTDR were almost estimated as considering the spatial resolution of $1 \mathrm{~m}$

Figures 5 and 6 show the calculated strain distributions in the undamaged and damaged yachts, respectively. As shown in Figs. 4 and 5, the sensing system with the BOTDR can provide almost accurate strain. On the other hand, as shown in Fig. 6, when the actual strain is sharply changing within the spatial resolution, it is estimated that the measured strain distribution won't show such large variation. This means that the strain variation with the high spatial frequency is removed from strain distributions in measurements using the BOTDR, and it is difficult to detect damage based on measuring variation of the Brillouin frequency shift. While the author proposed a method based on detecting shape variation of the Brillouin spectrum to find large variation in a strain distribution within the spatial resolution [14], it is essential that we make enough spatial resolution to detect the strain variation due to damage.

The spatial resolution of fiber-optic distributed sensors using Brillouin scattering, however, has been much improved notably by several research groups. Murayama et al. improved the spatial resolution of the commercially available BOTDR up 
to $0.3 \mathrm{~m}$ by the technique based on the fact that the profile of the Brillouin gain spectrum changes depending on strain distributions [21]. Sakairi et al. achieved the 20-cm resolution of the BOTDR [22], and Mizuno et al. demonstrated distributed measurements with the $6-\mathrm{mm}$ spatial resolution by using the Brillouin optical correlation domain reflectometry (BOCDR) [23]. The pulse-prepump BOTDR, different pulse-width pair Brillouin optical time domain analysis (BOTDA), and dark-pulse BOTDA are other examples [24-26]. Song et al. achieved the spatial resolution of $1.6 \mathrm{~mm}$ using Brillouin optical correlation domain analysis (BOCDA) [27]. However, the sensing system tends to be complicated for a higher resolution, and then it would result in higher costs and incomplete robustness.

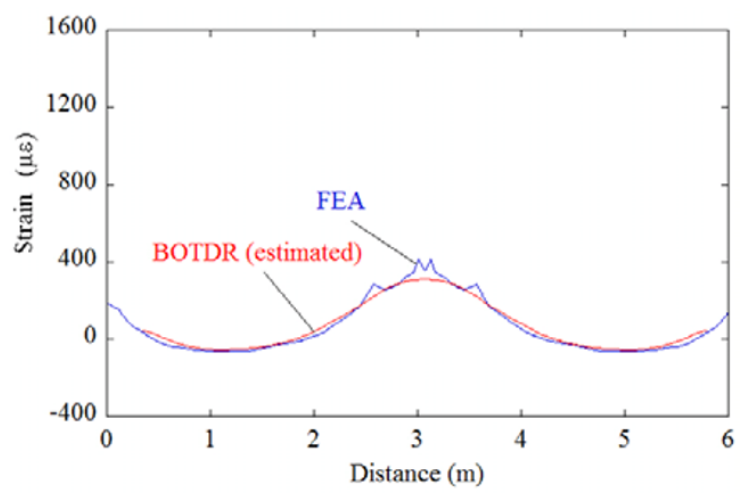

Fig. 5 Strain distributions without failure.

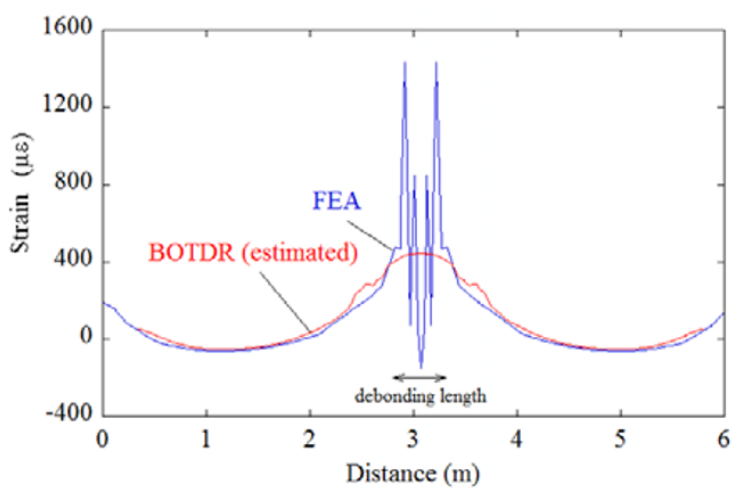

Fig. 6 Strain distributions with failure.

The authors have developed the sensing system with FBGs to measure the strain distribution with a high spatial resolution. While FBGs with some interrogation systems are ordinarily employed for quasi-distributed strain or temperature measurement, they can be also applied to fully-distributed sensing. In the next section, a technique to measure strain distributions along FBGs by using the optical frequency domain reflectometry (OFDR) is described.

\section{Distributed strain sensing with high spatial resolution}

In order to achieve distributed strain sensing with the high spatial resolution, we have developed the sensing system based on the OFDR. This section describes the principle of the sensing system and shows a measurement example.

\subsection{Principle of sensing system}

A typical system of the sensing system consists of a tunable laser source (TLS), photo detectors (PD), broadband reflectors (R), 3-dB couplers (C), a long-length FBG whose length is about $100 \mathrm{~mm}$, and a computer (PC) with a high-speed analog to digital (A/D) converter (DAQ). The schematic of the sensing system is shown in Fig. 7. The laser source inserts the coherent light and sweeps its wavelength. The lights split at $\mathrm{C} 1$ and propagate into two interferometers. Interferometer 1 generates an external clock of DAQ to acquire a signal of Interferometer 2 with a constant wavenumber interval. The signal of Interferometer $1, D_{1}$, is expressed as

$$
D_{1}=\cos \left(2 n_{\text {eff }} L_{R} k\right)
$$

where $n_{\text {eff }}$ is the effective refractive index of the optical fiber core, $L_{R}$ is the distance between $\mathrm{R} 1$ and $\mathrm{R} 2$, and $k$ is the wavenumber expressed as $k=2 \pi / \lambda$. By triggering $D_{1}$ at a constant value, we obtain the constant sampling interval given by

$$
\Delta k=\frac{\pi}{n_{\text {eff }} L_{R}} .
$$

The signal of Interferometer 2, $D_{2}$, which includes the information about the FBGs, is detected at PD 2 and sampled with the constant interval $\Delta k$ at DAQ. An approximated description of $D_{2}$ is often preferred for the purpose of the conceptual introduction, which is 


$$
D_{2}=\sum_{i} R_{i}(k) \cos \left(2 n_{\mathrm{eff}} L_{i} k\right)
$$

where $L_{i}$ is the distance between $\mathrm{R} 3$ and the $i$ th section of the FBG, and $R_{i}$ is the Bragg spectrum of the $i$ th section of the FBG. This indicates that Bragg spectra at each reflected location have the individual accompanying waves whose frequency corresponds to the distance between R3 and the reflected location. This approximated expression is valid when the reflectivity of the FBGs is low [28].

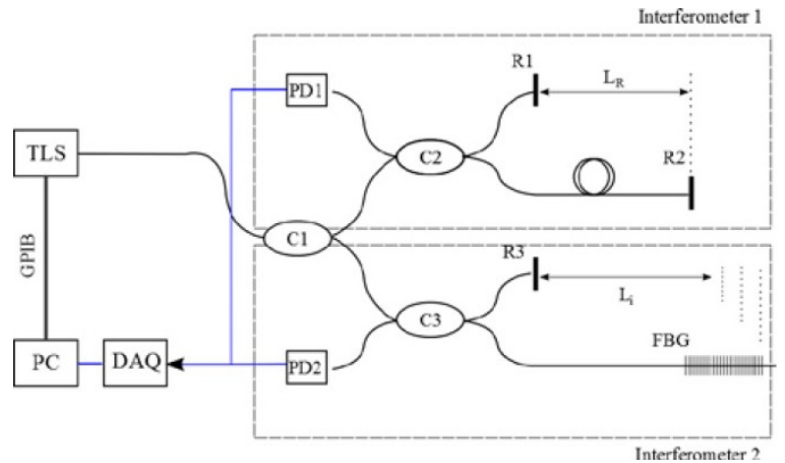

Fig. 7 Schematic of sensing system.

We applied the signal processing which was based on the short-time Fourier transform (STFT) to $D_{2}$. In this case, the time and frequency in the usual STFT corresponded to the wavenumber and frequency, respectively. A sliding window was used to extract a certain section of signal data as depicted in Fig. 8. The FFT was applied to the extracted data set with zero-padding, and a power profile was obtained as a function of the position. When the

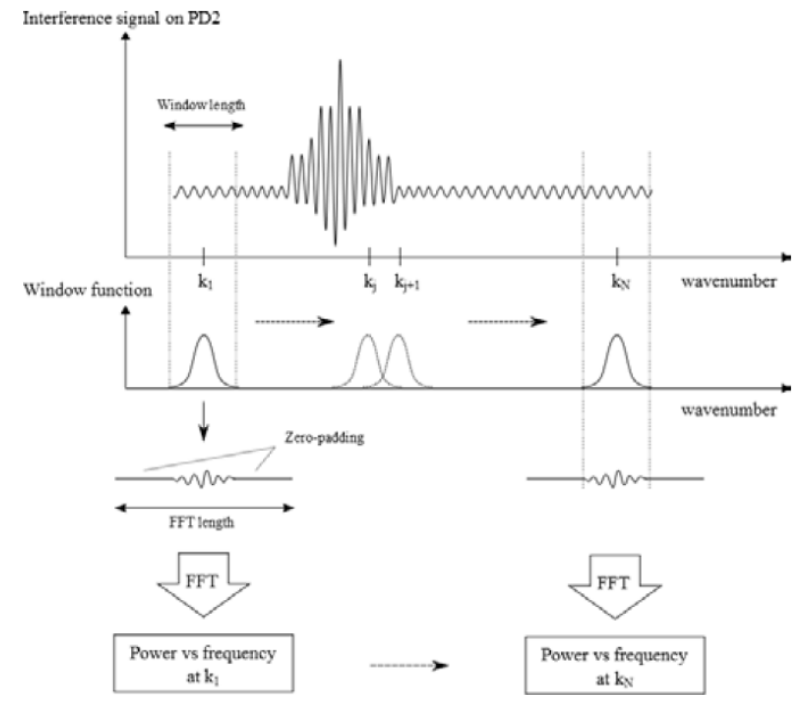

Fig. 8 Computing procedure of the signal processing. sliding window covered the whole range of the wavenumber, we obtained the power profile on the wavenumber-frequency two-dimensional coordinate. Converting the frequency into position, we could observe a Bragg spectral distribution on the graph called spectrogram.

\subsection{Measurement example}

We demonstrated a measurement of the $100-\mathrm{mm}$ FBG with the Bragg wavelength of $1549.4 \mathrm{~nm}$ using the sensing system based on the OFDR. The system parameters are listed in Table1. Figure 9 shows the obtained interference signal on PD2. It can be seen that the accompanying waves are superimposed on

Table 1 Geometrical and optical parameters.

\begin{tabular}{c|c}
\hline$L_{R}(\mathrm{~m})$ & 15.47 \\
\hline Wavelength sweep range of TLS $(\mathrm{nm})$ & $1548-1555$ \\
\hline Wavelength sweep speed of TLS $(\mathrm{nm} / \mathrm{s})$ & 10 \\
\hline$n_{\text {eff }}$ & 1.46 \\
\hline Window length (pm) & 400 \\
\hline FFT length (data points) & $2^{15}$ \\
\hline
\end{tabular}

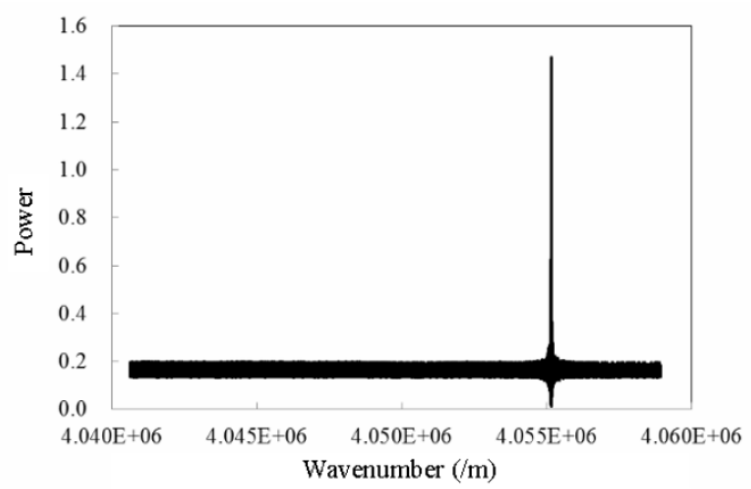

(a) Whole range of wavenumber

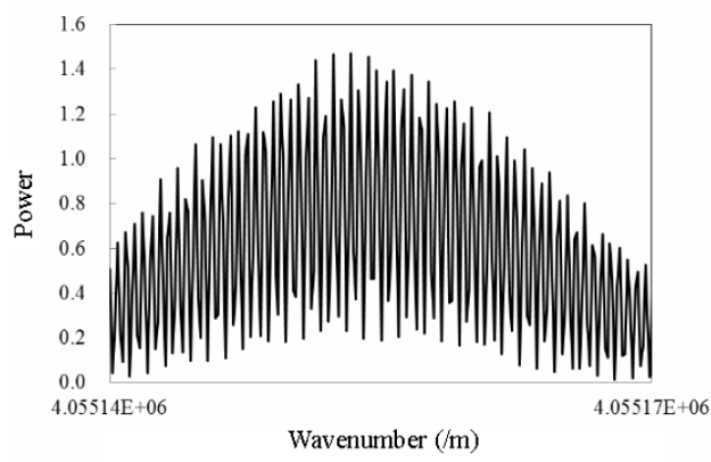

(b) Partial range of wavenumber

Fig. 9 Experimentally obtained interference signal form PD2. 
the profile of the Bragg spectrum. Figures 10, 11, and 12 are the spectrogram, the distribution of the Bragg wavelength, and the distribution of the spectral peak, respectively. The amount of every window slide for the STFT was adjusted so that the power profiles were plotted every $5.0 \mathrm{pm}$ in wavelength-wise in Fig. 10, whereas given the $L_{R}$ and FFT length resulted in the plot interval of $0.47 \mathrm{~mm}$ in position-wise. The Hanning window was used. The uniform distribution of the Bragg wavelength can be observed in Fig. 11. In this case, the spatial resolution of less than $1.0 \mathrm{~mm}$ can be achieved as described in Section 6.

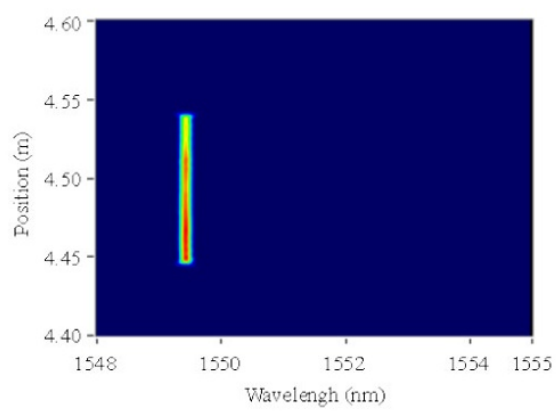

Fig. 10 Experimentally obtained spectrogram.

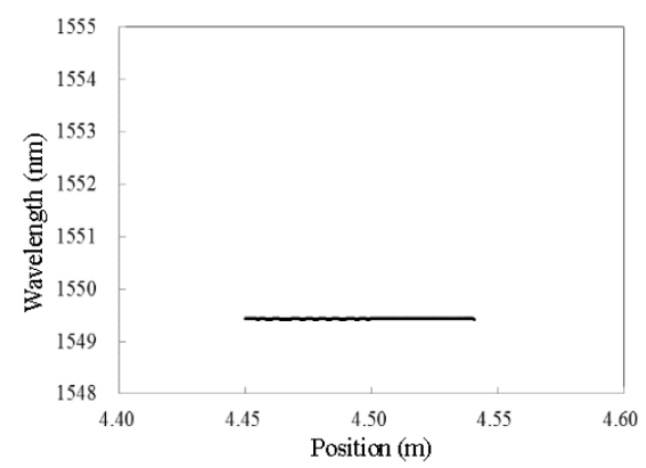

Fig. 11 Distribution of the Bragg wavelength.

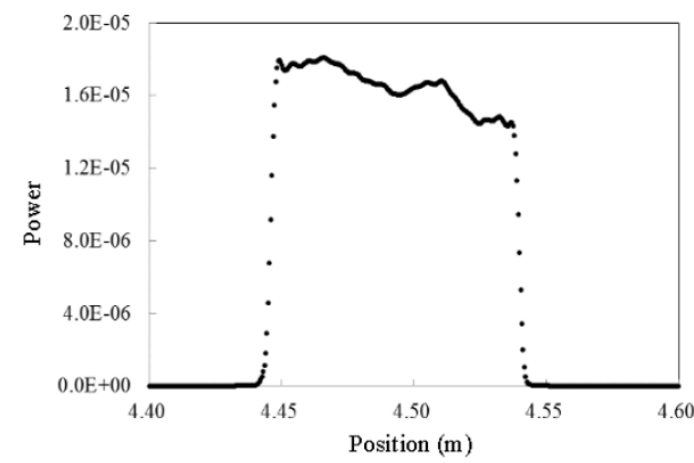

Fig. 12 Distribution of spectral peak.

\section{Damage detection in adhesive joint}

In this paper, we show an application of the distributed sensing system to strain monitoring and damage detection of an adhesive joint [16].

The use of the adhesive bonding in composites is recently increasing in aerospace, marine, and automotive sectors. Lap-shear joints are the most common configurations, and they have been extensively studied. Analytical research and numerical approaches to determine the stress or strain distribution inside the adhesive and at the interfaces between the adhesive and the adherends have been proposed by many researchers [29-31], since those are essential to find an optimized design which results in the higher strength and effectiveness in terms of the load-carrying capacity. The stress or strain distribution inside the adhesive layer can also provide the information on the quality in the manufacturing process and on the damage or degradation for in-service structures. By qualitative sensing techniques to determine stress or strain fields inside the adhesive layer along the overlap, it is expected that we can verify the manufacturing process and assess the structural integrity of joints.

In this section, the distributed strain measurements inside the adhesive of a single-lap joint and damage detection based on the measured strain distributions are shown.

\subsection{Specimen geometry and sensor installation}

The dimensions of the test specimens and the locations of the FBG sensors are shown in Fig. 13. The aluminum (A2017-T3) adherends were bonded Araldite $^{\circledR}$ 2011, two component epoxy paste, and the adhesive layer was controlled to be $1 \mathrm{~mm}$. Two long-length FBGs whose gauge length was about $80 \mathrm{~mm}$ were embedded into both sides of the adhesive layer of each single-lap joint as shown in Fig. 13. In FBG 1, the portion from $x=-30$ to 0 was bonded on the surface of the left adherend, the portion from $x=0$ to 30 was embedded inside the adhered section of the joint, and the portion from $x=$ 
30 to 50 was kept free. On the other hand, in FBG2, the portion from $x=-20$ to 0 was kept free, the portion from $x=0$ to 30 was embedded inside the adhered section, and the portion from $x=30$ to 60 was bonded on the surface of the right adherend. The portion in a free stress condition was used as the reference part for temperature compensation. FBGs were set on the V-shaped groove machined on the surface of the aluminum adherend to prevent FBGs from bending during the fabrication.

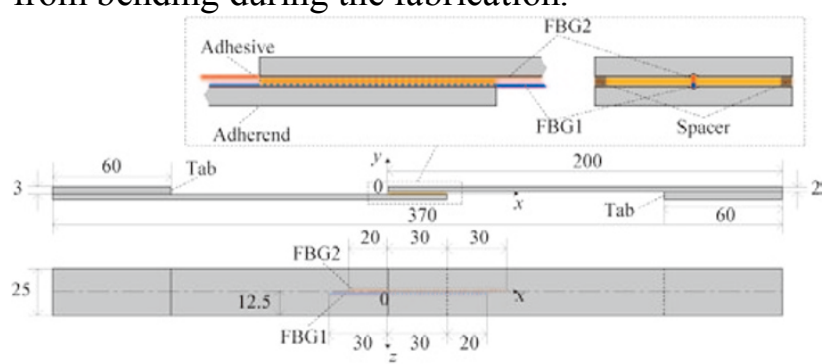

Fig. 13 Schematic of single-lap joint and FBG sensors (dimension in $\mathrm{mm}$ ).

\subsection{Strain measurements during static tensile test}

Static tensile loads were applied to the specimens by a universal test machine, and strain distributions along the FBG sensors were measured. The load cases in the test are shown in Fig. 14. The joint was broken at $6673 \mathrm{~N}$ during the load case of A11. Debonding at the substrate-adhesive interface was major failure mode, and a number of acoustic emission (AE) events were detected by the $\mathrm{AE}$ sensor (Physical Acoustic Corporation) as shown in Fig. 14.

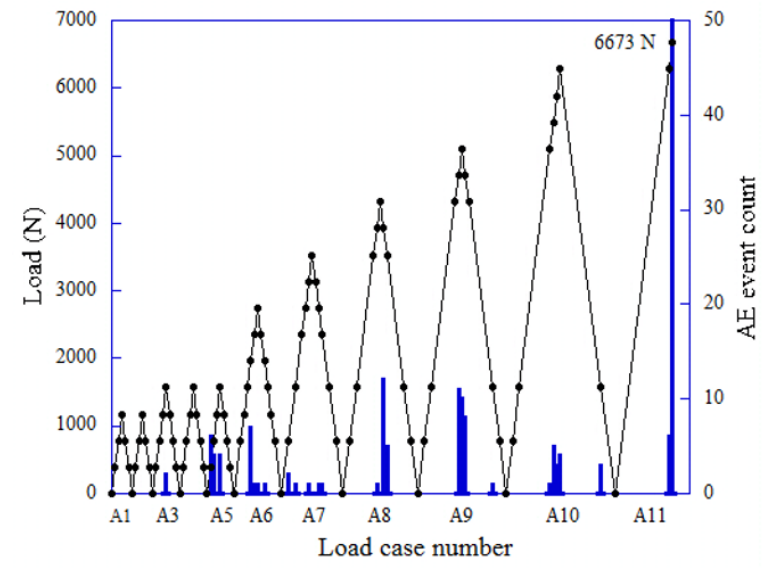

Fig. 14 Load cases and AE event count in the tensile test.
Figures 15(a) and 15(b) show strain distributions measured by FBGs and calculated by FEA at the maximum loads in the load cases of A3 and A8. The agreement between the measured strains and the calculated ones (adherend) is excellent in A3 and A8. Such quantitative strain distributions inside the adhesive layer had not been obtained by means other than fiber-optic distributed sensors with the high spatial resolution.

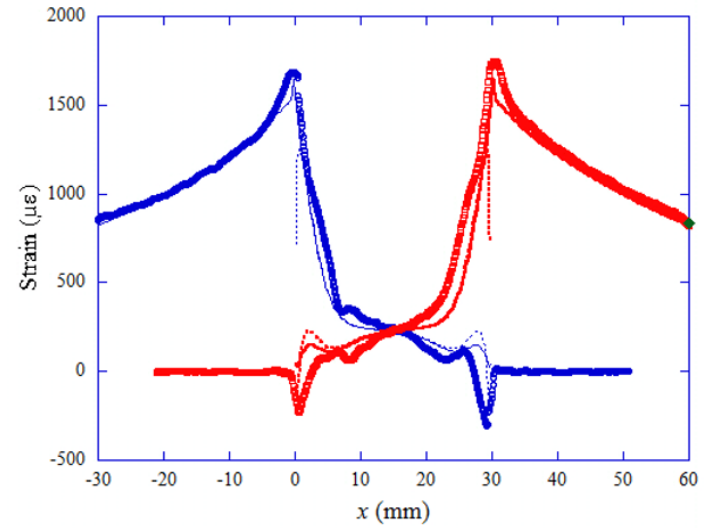

(a) $1570 \mathrm{~N}$ in $\mathrm{A} 3$

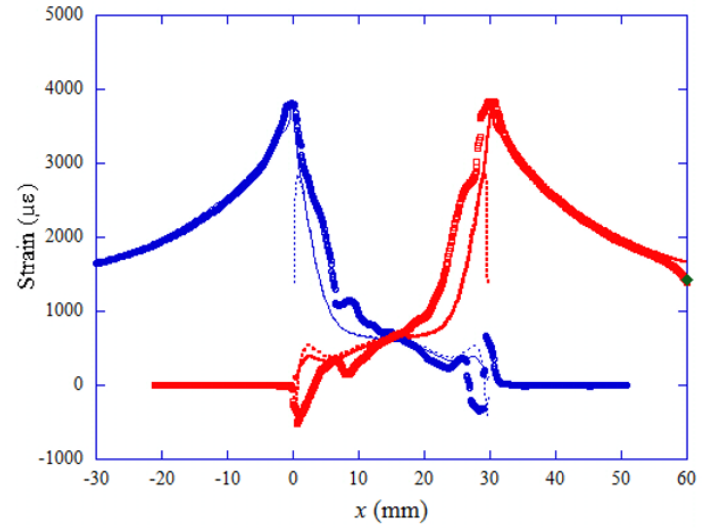

(b) $4316 \mathrm{~N}$ in $\mathrm{A} 8$

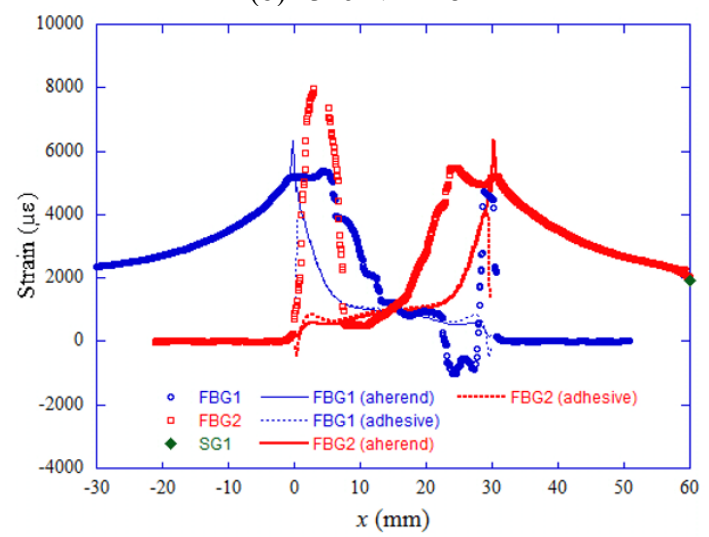

(c) $6671 \mathrm{~N}$ in $\mathrm{A} 11$

Fig. 15 Strain distributions obtained from experimental and numerical analysis. 


\subsection{Damage detection of single-lap joint}

As shown in Fig. 15(c), however, the measured strain distribution changed in A11, as if the overlap length had been shorter. It might imply the debonding occurred at the ends of the overlap. Therefore, the interfacial failure was introduced to the interface between the adherend and the adhesive at both ends of the overlap in the FE model. The debonding regions were from $x=0$ to 5 at $y=1$ and from $x=25$ to 30 at $y=0$. In the width direction, the debonding was penetrated. Figure 16 shows the calculated strain distribution when the single-lap joint with the debonding was subjected to the tensile load of $6278 \mathrm{~N}$ in A10 as well as the measured strain distribution. It can be seen that each FBG measured strain on the adherend at the interface without the debonding, and on the other hand, they were peeled off from the adherend to measure the strain on the adhesive at the interface with the debonding.

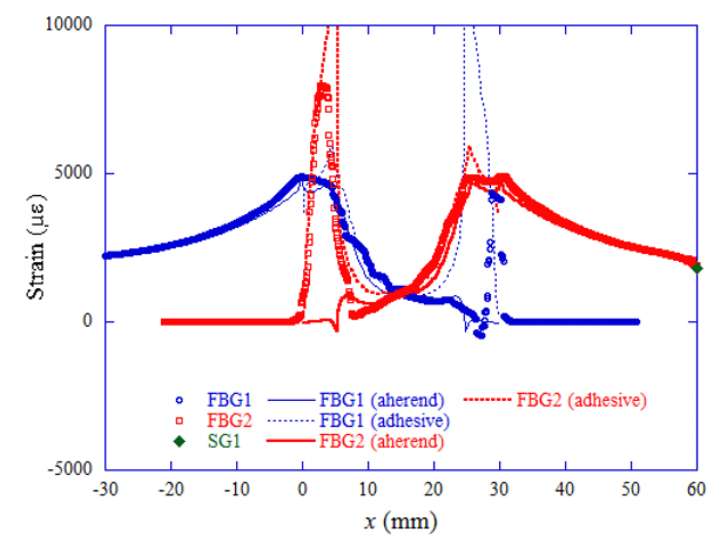

Fig. 16 Strain distributions with the debonding at the ends of the overlap at the load of $6278 \mathrm{~N}$ in A10.

Figure 17 shows the strain distributions measured at about $1.5 \mathrm{kN}$ in the four load cases. The strain distributions in the adhesive layer changed gradually, and this shows damage occurrence or propagation. From comprehensive data analysis, there were several remarkable changes in the strain distributions of $\mathrm{A} 8$ and $\mathrm{A} 9$ in which a lot of $\mathrm{AE}$ were detected by $\mathrm{AE}$ sensors, as shown in Fig. 14. It is expected that more detailed analysis of the measured strain distributions can provide the geometric information on the failure and failure mode.

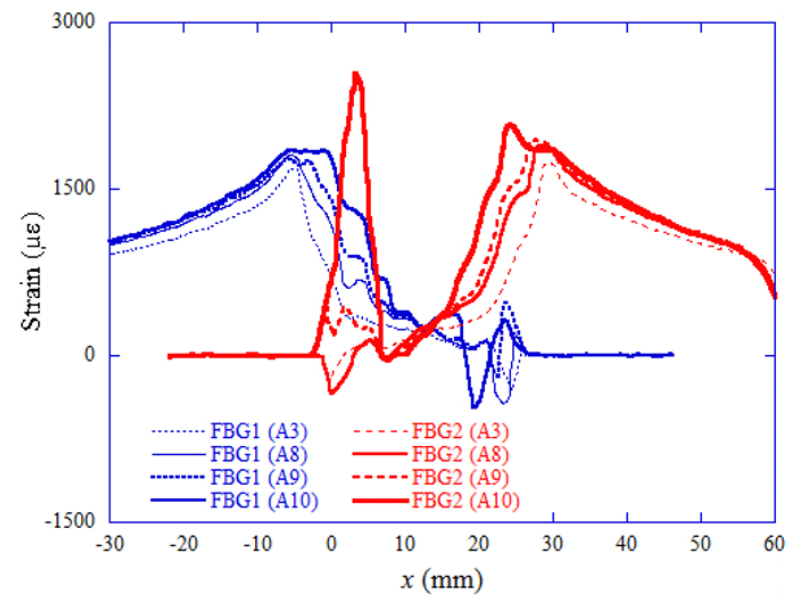

Fig. 17 Measured strain distributions at the load of about $1.5 \mathrm{kN}$ in $\mathrm{A} 3, \mathrm{~A} 8, \mathrm{~A} 9$, and $\mathrm{A} 10$.

The authors also applied the fiber-optic distributed sensors to defect detection in welded joints and confirmed that an anomalous distortion due to welding defect was found in the measured strain distribution [32].

\section{Load identification}

Distributed loads of the wings of an in-service aircraft can influence on the in-flight safety and fatigue aging. Therefore, if we know the loads of the in-service aircraft, we can increase the in-flight safety and the efficiency of the maintenance inspection for the aircraft which is designed based on the damage tolerance. However, in general, the in-flight load distribution of a wing cannot be measured directly by sensors. It can be predicted by other structural conditions, such as the strain and displacement, and this problem should be solved by an inverse analysis [33-35].

The authors have developed the prediction technique of the distributed loads on a structure based on strain distributions measured by fiber-optic sensors $[2,17,36]$. In this paper, load identification of a simply supported aluminum beam with FBGs is shown. 


\subsection{Distributed strain measurement of simply supported beam}

The dimensions of the test beam and locations of the sensors are shown in Fig. 18. The test beam had the length of $2000 \mathrm{~mm}$ and the rectangular hollow section, the height, width and thickness of which were $15 \mathrm{~mm}, 30 \mathrm{~mm}$ and $1.5 \mathrm{~mm}$, respectively. The test beam was made of aluminum alloy. A 1500-mm gauge length sensor with FBGs was located along the central line of the upper surface and bonded with the epoxy adhesive (Araldite ${ }^{\circledR}$ 2011). The FBGs were fabricated by a conventional exposure method using a $\mathrm{KrF}$ excimer laser and a uniform period mask. Since the length of the mask was $100 \mathrm{~mm}$, every $100-\mathrm{mm}$ range was exposed 15 times. The distance between two simply supported ends was $1600 \mathrm{~mm}$. As a reference, three strain gauges were bonded along the FBGs. The test beam was subjected to concentrated static loads with wires, hooks, and weights.

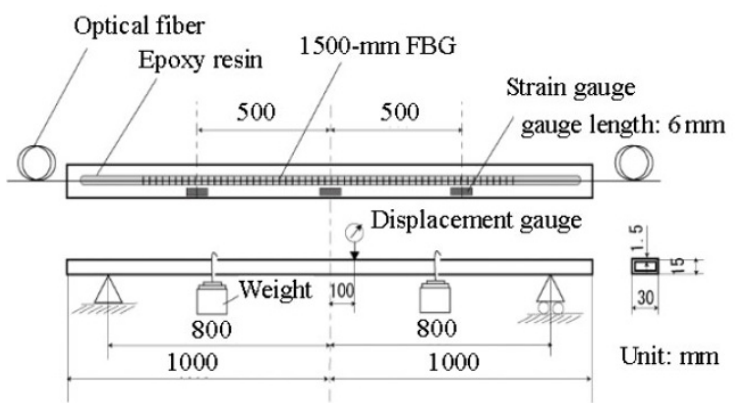

Fig. 18 Dimensions of the test beam and locations of the sensors.

Figure 19 shows the measurement strain distributions under two load cases. Load points and load values are also shown in the figure. The origin of the $x$-axis shows the center of the beam, the dots show the results of measurement by the FBGs, the circles show measurement results by the three strain gauges, and the solid lines show the theoretical solutions of the conventional beam equation. It can be seen that the $1500-\mathrm{mm}$ gauge length sensor is able to measure the strain distribution of the test beam accurately in both load cases.

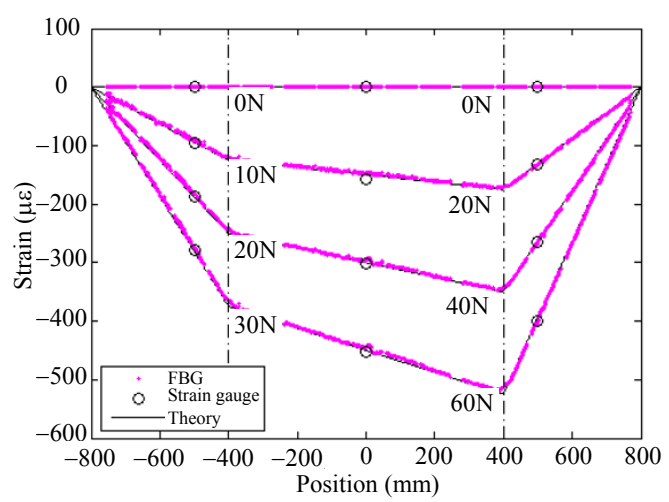

(a) Load case 1

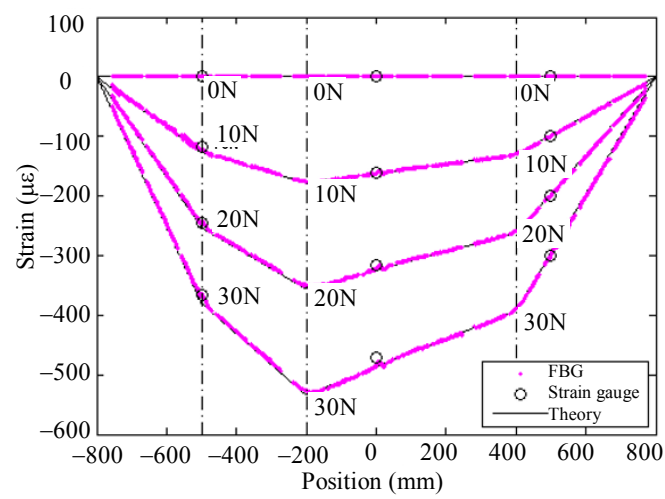

(b) Load case 2

Fig. 19 Strain distributions of the test beam.

\subsection{Load identification from measured strain data}

Considering the linear elastic problem, measured strains can be expressed as

$$
\varepsilon_{j}^{m}=\sum_{i=1}^{n} s_{j i} p_{i}, \quad j=1,2, \cdots, m
$$

where $p_{i}(i=1,2, \cdots, n)$ is the assumed distributed load at each node, and $s_{j i}$ is the $j$-th strain value when the unit load $p_{i}=1$ acts at the $i$-th node and can be calculated a priori by the FEA or other analytical methods [35].

Then, the distributed load can be identified from the measured strain data by solving the (4) as

$$
p_{i}=\sum_{j=1}^{m_{s}} s_{i j}^{+} \varepsilon_{j}^{m}, \quad i=1,2, \cdots, n .
$$

Since generally $m_{i} \neq n$, the matrix $\left[s_{j i}\right]$ is a non-square matrix. Therefore, $\left[s_{i j}^{+}\right]$should be a pseudo-inverse matrix. In this study, the Moore-Penrose pseudo-inverse matrix is used as $\left[s_{i j}^{+}\right]$. 
In this paper, the number of assumed load nodes $n=300$, and the number of measured strains $m_{s}=$ 1408. In calculating the Moore-Penrose pseudoinverse matrix, small singular values of the matrix $\left[s_{j i}\right]$ may result in the divergence. It is known that this divergence problem can be avoided by replacing such small singular values with zero, that is, by reducing the rank of matrix. In this paper, we choose 30 as the rank of the matrix. Then the condition number, which is the ration of the maximum singular value to the minimum non-zero singular value, was 894.9.

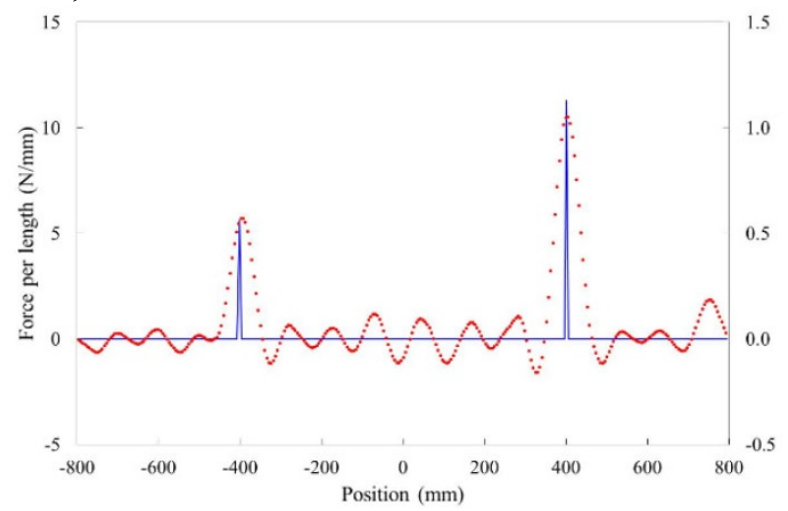

(a) Load distributions

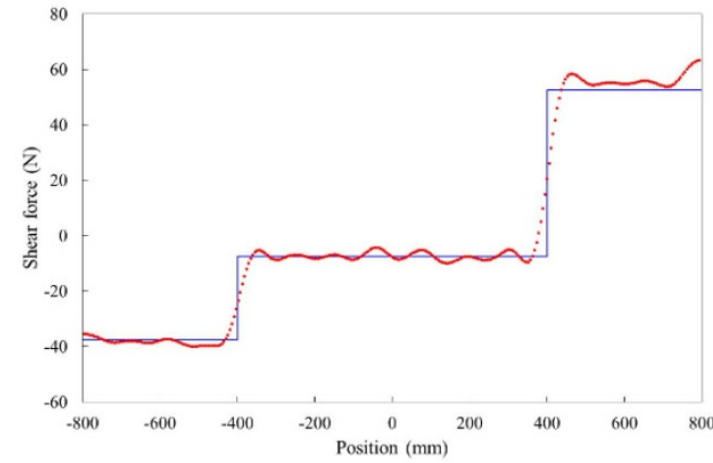

(b) Shearing force diagram (S.F.D.)

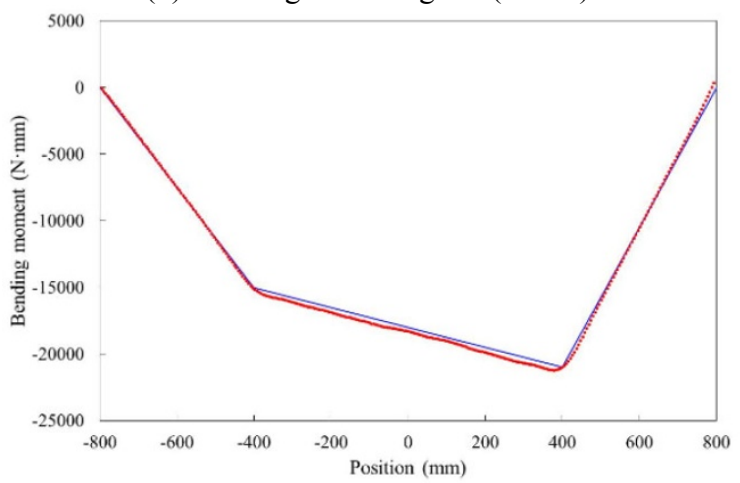

(c) Bending moment diagram (B.M.D)

Fig. 20 Load identification for load case 1.
Figures 20 and 21 show load identification results from the measured strain data for the two load cases. Figures 20(a) and 21(a) show distributed loads that are the forces per length for load cases 1 and 2, respectively. Solid line is actual load applied the beam, and solid circle shows the estimated load. Figures 20(b) and 21(b) show shearing force distributions that are given as the integral of the load distributions. In addition, Figures 20(c) and 21(c) show bending moment distributions that are given as

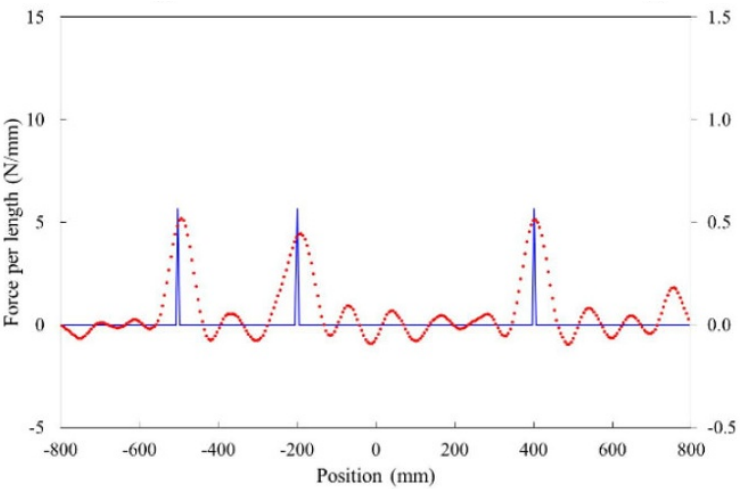

(a) Load distributions

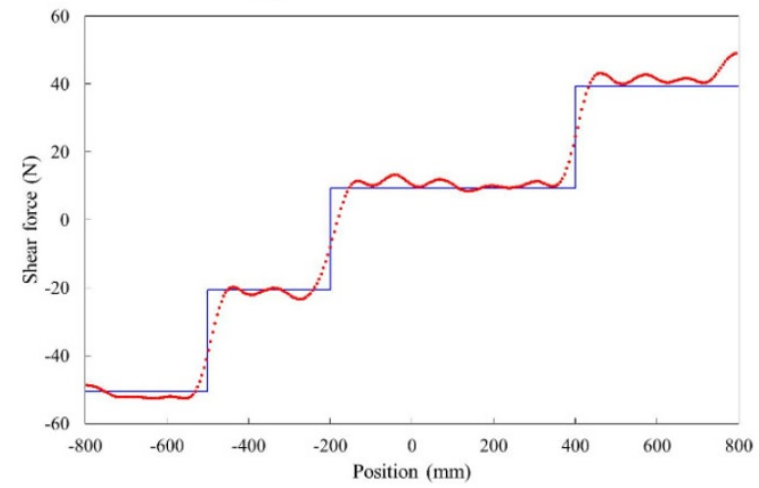

(b) Shearing force diagram (S.F.D.)

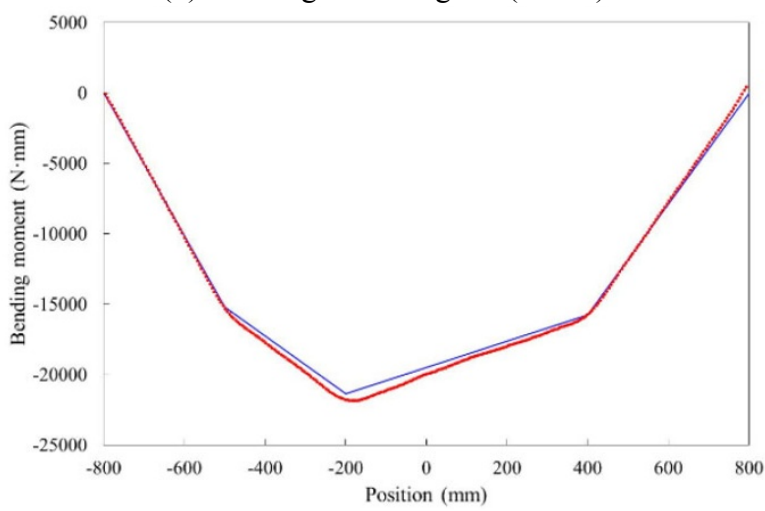

(c) Bending moment diagram (B.M.D)

Fig. 21 Load identification for load case 2. 
the integral of the shearing force distributions. It can be seen that the shearing force and bending moment could be identified precisely.

The authors also applied this prediction technique with the FEA to load identification of the 6-m composite wing structure. FBG arrays including 246FBGs were equipped with the wing, and the load applied to the wing in structural tests could be estimated [17].

\section{Improvement of applicability of the distributed sensing system}

In this section, the fundamental studies to improve the applicability of the fiber-optic distributed strain sensors to SHM based on strain monitoring are shown.

\subsection{Development of sensing system based on simulation}

In order to evaluate the sensing capability of the fiber-optic distributed strain sensing system and assess its applicability for various purposes of structural monitoring, the estimation of its theoretical performance is important. When measurements using the sensing system are simulated, there are a number of factors which influence the measurement results. Parameters which are used in the signal processing such as the window length influence the measurement accuracy and spatial resolution. Geometric dimensions of optical fiber lengths in the interferometers of the OFDR determine the sensing range, and polarization states of propagating lights in the fibers influence the spectral profile of the obtained signal. Furthermore, installation methods of FBGs such as embedding or bonding and locations of FBGs influence the response of the optical signal against the structural parameters of interest. Consequently, it can be said that the output of the sensing system is the result of interactions between these factors. As an estimation method of the theoretical performance, we introduce a form of simulation analysis which can consider the various influencing factors in a single sequence of simulations.

This simulation analysis mainly consists of four steps, which are loading simulation for calculating the stress/strain conditions in optical fibers, photo-elastic effect analysis for calculating the variation of the optical properties, OFDR optical simulation for producing the interfered light signal, and signal processing for demodulating the signal. Structural parameters of interest such as strain distributions are transferred to FBGs. They influence the optical property of the FBGs. The propagating lights in the optical fiber which experience the optical property variations are interfered and detected in the sensing system. The detected signal is demodulated into a form of strain distributions through signal processing. We conduct the simulation analysis by seamlessly concatenating these processes of the four steps.

\subsubsection{Simulation method}

We introduced the sequence of the simulation analysis by taking an example of the strain concentration measurement of an aluminum plate with holes under tensile loads [37]. The schematic of the aluminum plate are depicted in Fig. 22. The longitudinal (horizontal) direction was set as $z$-axis and the vertical direction $x$-axis. $y$-axis was in the direction of the thickness. The dimensions of the aluminum plate were $250 \mathrm{~mm} \times 30 \mathrm{~mm} \times 2 \mathrm{~mm}$. Three holes were located to touch the central line of the width of the plate. Diameters of them were $2 \mathrm{~mm}$, $2 \mathrm{~mm}$, and $4 \mathrm{~mm}$, respectively, and they had $15-\mathrm{mm}$ interval between each. A 50-mm FBG was assumed to be bonded $0.5 \mathrm{~mm}$ away from the central line. Tensile loads were applied in the $z$ direction.

\section{A. Loading simulation}

We often use FEA for loading simulation to model and simulate mechanical conditions of target structures. In this example, we modeled the aluminum plate with the holes as shown Fig. 22. We did not consider the existence of the FBG, however, in some cases such as when FBGs were embedded into composites, and the effect of stress transfer 
from surrounding material on FBGs were not negligible, the FE model might need to include the FBGs themselves.

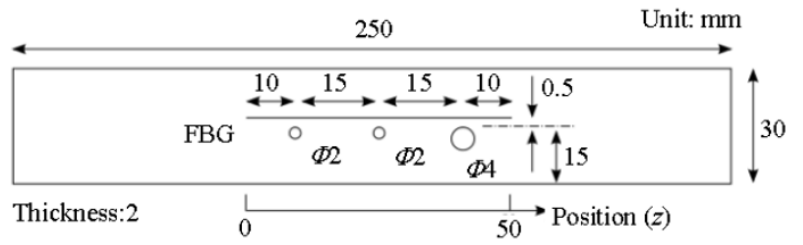

Fig. 22 Schematic of the aluminum plate with holes.

The strain distribution was calculated along the location of the FBG when the tensile load of $300 \mathrm{kgf}$ was applied to the aluminum plate. The Young's modulus of the aluminum plate was set as $70.3 \mathrm{GPa}$ and Poisson's ratio as 0.345 . We assumed that the longitudinal strain, $z$-strain, of the plate was identical to those of the FBG. Stresses in the cross section of the FBG were assumed to be zero.

\section{B. Photo-elastic effect analysis}

In general, stresses within an FBG affect the refractive indices of the two polarization modes and the grating period and therefore cause variations of the Bragg wavelengths [38, 39]. Bragg wavelength shifts in accordance with the two polarization modes, $\Delta \lambda_{B x}$ and $\Delta \lambda_{B y}$, are calculated based on the photo-elastic effect using the stresses, $\sigma_{x}, \sigma_{y}$, and $\sigma_{z}$ as

$$
\left\{\begin{array}{l}
\Delta \lambda_{B x}=-\frac{n_{\mathrm{eff}}^{3} \Lambda}{E}\left\{\left(P_{11}-2 v P_{12}\right) \sigma_{x}+\left[(1-v) P_{12}-v P_{11}\right]\left(\sigma_{y}+\sigma_{z}\right)\right\}+\frac{2 n_{\mathrm{eff}} \Lambda}{E}\left[\sigma_{z}-v\left(\sigma_{x}+\sigma_{y}\right)\right] \\
\Delta \lambda_{B y}=-\frac{n_{\mathrm{eff}}^{3} \Lambda}{E}\left\{\left(P_{11}-2 v P_{12}\right) \sigma_{y}+\left[(1-v) P_{12}-v P_{11}\right]\left(\sigma_{x}+\sigma_{z}\right)\right\}+\frac{2 n_{\mathrm{eff}} \Lambda}{E}\left[\sigma_{z}-v\left(\sigma_{x}+\sigma_{y}\right)\right]
\end{array}\right.
$$

where $E$ is the Young's modulus of the fiber core, $n_{\text {eff }}$ is the effective refractive index of the FBG, $\Lambda$ is the grating pitch, $P_{11}$ and $P_{12}$ are the photoelastic coefficients, and $v$ is the Poisson's ratio of the fiber core [40]. In this example, we assumed

$$
\left\{\begin{array}{l}
\sigma_{x}=0 \\
\sigma_{y}=0 \\
\sigma_{z}=E \varepsilon_{z}(z)
\end{array}\right.
$$

where $\varepsilon_{z}$ is $z$-strain of the FBGs calculated by the previous loading simulation. In this case, Bragg wavelengths are identical in both polarization modes Consequently, we obtained Bragg wavelength shifts at arbitrary locations within the FBG.

\section{OFDR optical simulation}

Here, the mode coupling of propagation lights is discussed in a single polarization mode because birefringence does not exist in this example. Corresponding simulations considering the birefringence are referred to the work of the authors [38].

The interfered light signal of the sensing system is described by

$$
I=\left|R L_{R}+R L_{\mathrm{FBG}}\right|^{2}
$$

where $R L_{R}$ and $R L_{\mathrm{FBG}}$ are reflected lights from $\mathrm{R} 3$ and the FBG, respectively. The reflected light from $\mathrm{R} 3$ is given by

$$
R L_{R}=e^{i\left(2 n z_{0} k+\pi\right)}
$$

where $n$ is the refractive index, and $z_{0}$ is the path difference between R3 and PD2 as depicted in Fig. 7 .

On the other hand, Interferometer 2 is modeled based on the piecewise-uniform approach using transfer matrices [42, 43]. We divide the optical fiber paths into multiple uniform sections and assign transfer matrices. The input and output relationship of the interferometer is expressed as

$$
\left[\begin{array}{l}
R(0) \\
S(0)
\end{array}\right]=\mathbf{T}\left[\begin{array}{l}
R\left(z_{2}\right) \\
S\left(z_{2}\right)
\end{array}\right]
$$

where $R(\mathrm{z})$ and $S(\mathrm{z})$ are the amplitudes of the forward and backward propagating modes, respectively, and $\mathbf{T}$ is the integrated transfer matrix. $\mathbf{T}$ is considered as a multiplication of transfer matrices of phases, $\mathbf{P}$, and individual sections of FBGs, $\mathbf{F}$, and is given by

$$
\mathbf{T}=\mathbf{P} \cdot \mathbf{F}_{(N)} \cdot \ldots \cdot \mathbf{F}_{(2)} \cdot \mathbf{F}_{(1)}
$$

where

$$
\mathbf{P}=\left[\begin{array}{cc}
\exp \left(-i n z_{1} k\right) & 0 \\
0 & \exp \left(i n z_{1} k\right)
\end{array}\right]
$$




$$
\mathbf{F}=\left[\begin{array}{cc}
\cosh \left(\gamma_{B} \Delta z\right)-i \frac{\hat{\sigma}}{\gamma_{B}} \sinh \left(\gamma_{B} \Delta z\right) & -i \frac{\kappa}{\gamma_{B j}} \sinh \left(\gamma_{B j} \Delta z\right) \\
i \frac{\kappa}{\gamma_{B}} \sinh \left(\gamma_{B} \Delta z\right) & \cosh \left(\gamma_{B} \Delta z\right)+i \frac{\hat{\sigma}}{\gamma_{B}} \sinh \left(\gamma_{B} \Delta z\right)
\end{array}\right]
$$

where $\Delta z$ is the section length, $\gamma_{B}$ is the mode-coupling coefficient, which is expressed using the general "dc" self-coupling coefficient $\hat{\sigma}$ and the "AC" coupling coefficient $\kappa$, as

$$
\gamma_{B}=\sqrt{\kappa^{2}-\hat{\sigma}^{2}}
$$

$\kappa$ is described as

$$
\kappa=\frac{k}{2} v \overline{\delta n}_{\text {eff }}
$$

where $v$ is the fringe visibility of the index change, and $\overline{\delta n}_{\text {eff }}$ is the "dc" index change spatially averaged over a grating period. $\hat{\sigma}$ is described as

$$
\hat{\sigma}=n\left(k-\frac{2 \pi}{\lambda_{B}}\right)+\sigma
$$

where $\lambda_{B}$ is the designed Bragg wavelength, and $\sigma$ is the " $\mathrm{dc}$ " coupling coefficient expressed as

$$
\sigma=k \overline{\delta n}_{\text {eff }} \text {. }
$$

When boundary conditions which are expressed as

$$
\begin{aligned}
& R(0)=1 \\
& S\left(z_{2}\right)=0
\end{aligned}
$$

are applied, $R L_{\mathrm{FBG}}$ is calculated as

$$
R L_{\mathrm{FBG}}=\frac{T_{21}}{T_{11}} .
$$

Consequently, the interfered light signal is obtained by (8).

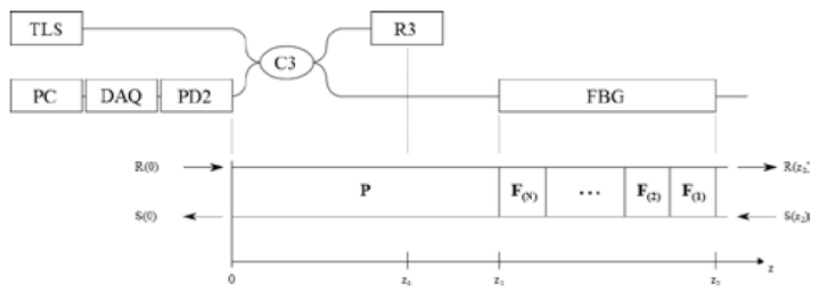

Fig. 23 Model of the interferometer for the numerical simulation.

\section{Signal processing}

We applied signal processing based on the STFT to the obtained signal $I$ in order to obtain the distributions of reflected Bragg spectra. The
Hanning window with the length of $400 \mathrm{pm}$ was used, which resulted in the spatial resolution of $0.9 \mathrm{~mm}$ as calculated in Section 6.1.3. The window slide was adjusted to achieve the 5.0-pm resolution of the wavelength. We calculated the strain distribution from the Bragg wavelengths shifts.

\subsubsection{Simulation result of aluminum plate with holes}

Figure 24 shows the strain distributions calculated by the FEA and by the simulation developed. It can be seen that the theoretical performance of the sensing system is applicable to detect the strain variations due to the existence of the holes. We also conducted a corresponding experiment using a polyimide-coated FBG with the length of $100 \mathrm{~mm}$. A portion of the FBG, whose length was about $50 \mathrm{~mm}$, was bonded to the surface of the aluminum plate. The experimental and calculated results of strain distribution measurements are in good agreement as seen in Fig. 24 . This indicates the validity and reliability of the simulation analysis. The simulation is expected to be utilized for various purposes such as determination of effective sensor locations, optimization of signal processing, investigation into the cause of the unexpected noise.

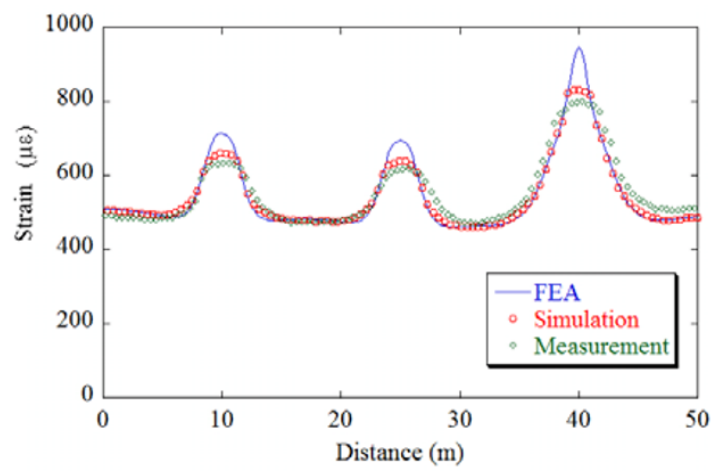

Fig. 24 Strain distributions of the aluminum plate with holes. 


\subsubsection{Discussions about spatial resolution based on simulation analysis}

It is no longer easy to experimentally find the spatial resolution of the fiber-optic distributed strain sensor with the high spatial resolution of less than $1 \mathrm{~mm}$. The authors have proposed the simulationbased investigation for determination of the spatial resolution.

In this paper, we discriminate between the spatial resolution and the spatial interval of distribution plots. The spatial interval is controlled by the FFT length. The longer length of the FFT produces larger amounts of data points in spatial distributions, which results in the smaller spatial interval (finer spatial distributions). Zero padding can adjust the FFT length as long as the calculation cost allows, therefore, the spatial distributions can be finer infinitely in theory. However, the sensitivity against strain variations, the spatial resolution, is dominated by the window length. Longer window lengths result in higher spatial resolutions and less sensitivity to the wavelength as trade-off.

The simulation analysis allows us to investigate the effect of the window length on the spatial resolution. We simulated a $100-\mathrm{mm}$ FBG signal in which the Bragg wavelength was shifted by $1.0 \mathrm{~nm}$ at its center using the piece-wise uniform approach. The FBG length was divided into 1000 sections, and the Bragg wavelengths of $1553 \mathrm{~nm}$ and $1554 \mathrm{~nm}$ were assigned to the sections corresponding to the first $50 \mathrm{~mm}$ and the rest part of the FBG, respectively. Other parameters used for the simulation were the same as listed in Table2. The Hanning window was used for the FFT. The FFT length was adjusted to $65536\left(2^{15}\right)$ points of data by zero-padding, which resulted in the spatial interval of $0.24 \mathrm{~mm}$. The amount of the window slides were adjusted so that the plot interval in the wavelength was $5.0 \mathrm{pm}$.

Figure 25 shows the spectrograms and the Bragg wavelength distributions of the 100-mm FBG with
Table 2 Parameters for OFDR optical simulation.

\begin{tabular}{cc}
\hline Parameters & Value \\
\hline$n$ & 1.46 \\
$v$ (visibility) & 0.394 \\
$\kappa$ & $1.00 \mathrm{~m}^{-1}$ \\
$N$ (number of sections) & 281 \\
$z_{0}$ & $1 \mathrm{~m}$ \\
$z_{1}-z_{0}$ (FBG location) & $4.200 \mathrm{~m}$ \\
$L_{R}$ & $15.47 \mathrm{~m}$ \\
Sweep range of laser source & $1552.0-1555.0 \mathrm{~nm}$ \\
Initial Bragg wavelength & $1552.5 \mathrm{~nm}$ \\
\hline
\end{tabular}

the $1.0-\mathrm{nm}$ shifts. Window lengths of 3752,7502 and 11252 points of data, which corresponded to $200 \mathrm{pm}, 400 \mathrm{pm}$, and $600 \mathrm{pm}$, respectively, were applied. The Bragg wavelengths were defined as the center of the full width at half maximum (FWHM) of individual spectra in the spectrograms. We defined the spatial resolution as the distance between the points at which the Bragg shift reached at $10 \%$ and $90 \%$ of the maximum. Bragg wavelength distributions in Fig. 25 show that the window length of $200 \mathrm{pm}, 400 \mathrm{pm}$, and $600 \mathrm{pm}$ achieved the spatial resolution of $1.6 \mathrm{~mm}, 0.9 \mathrm{~mm}$, and $0.6 \mathrm{~mm}$. On the other hand, the wavelength resolution decreased as the window length increased. This can be seen in the spectrograms in Fig. 25. The width of the spectra was broadened when the longer window length was applied. The root-mean-square (RMS) values of the Bragg wavelength calculation error in the sections $0 \mathrm{~mm}-48 \mathrm{~mm}$ and $52 \mathrm{~mm}-$ $100 \mathrm{~mm}$, which excluded the FBG central section, were $3.4 \mathrm{pm}, 3.2 \mathrm{pm}$, and $1.6 \mathrm{pm}$ for the window length of $200 \mathrm{pm}, 400 \mathrm{pm}$, and $600 \mathrm{pm}$, respectively. Considering the wavelength plot interval of $5.0 \mathrm{pm}$, this indicates that the Bragg wavelength defined by the center of FWHM is not affected by the window length. 

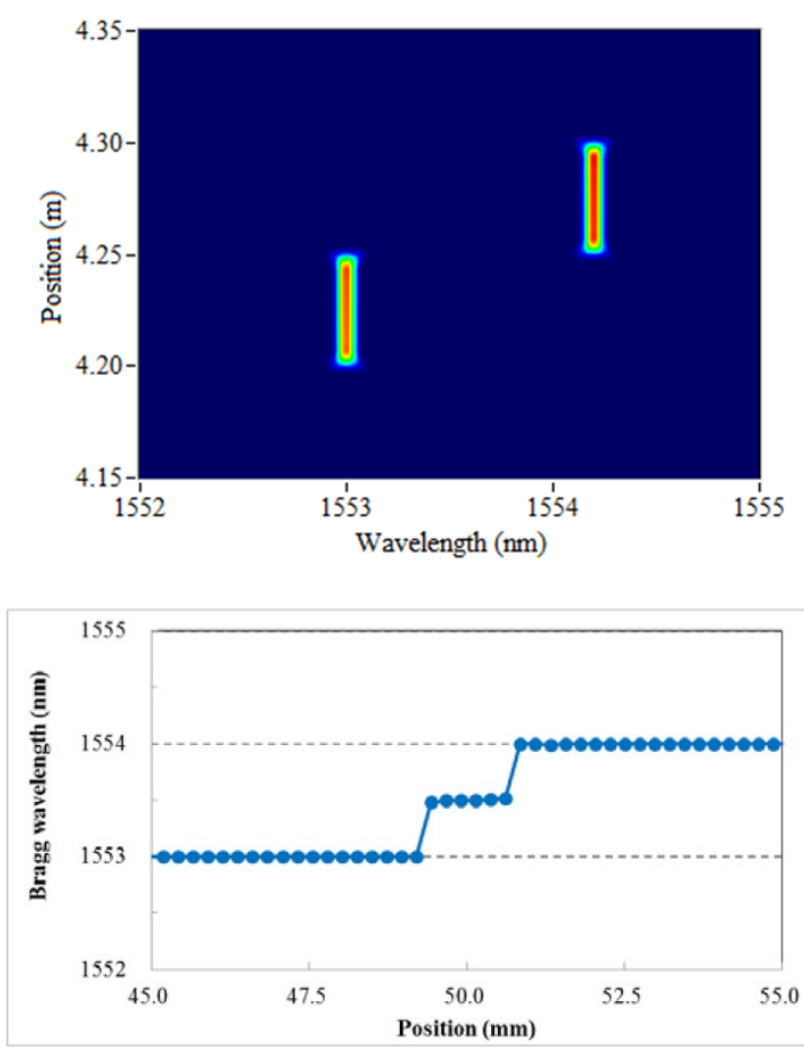

(a) Window length $=200 \mathrm{pm}$
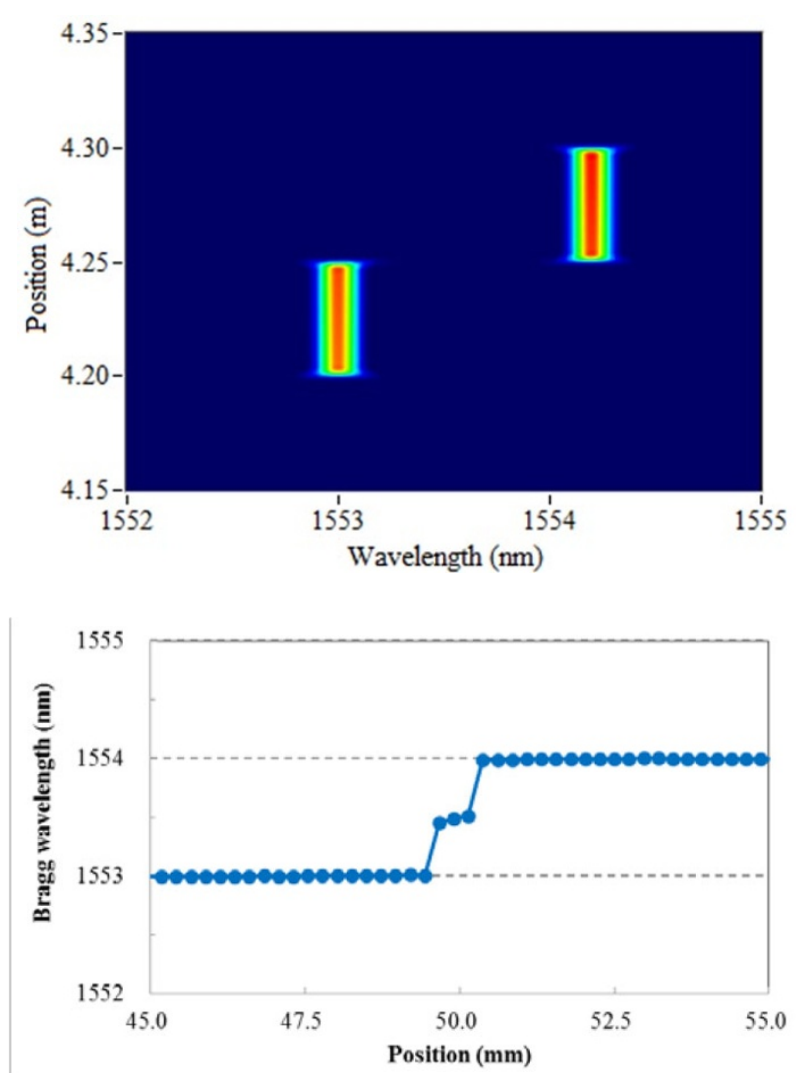

(b) Window length $=400 \mathrm{pm}$
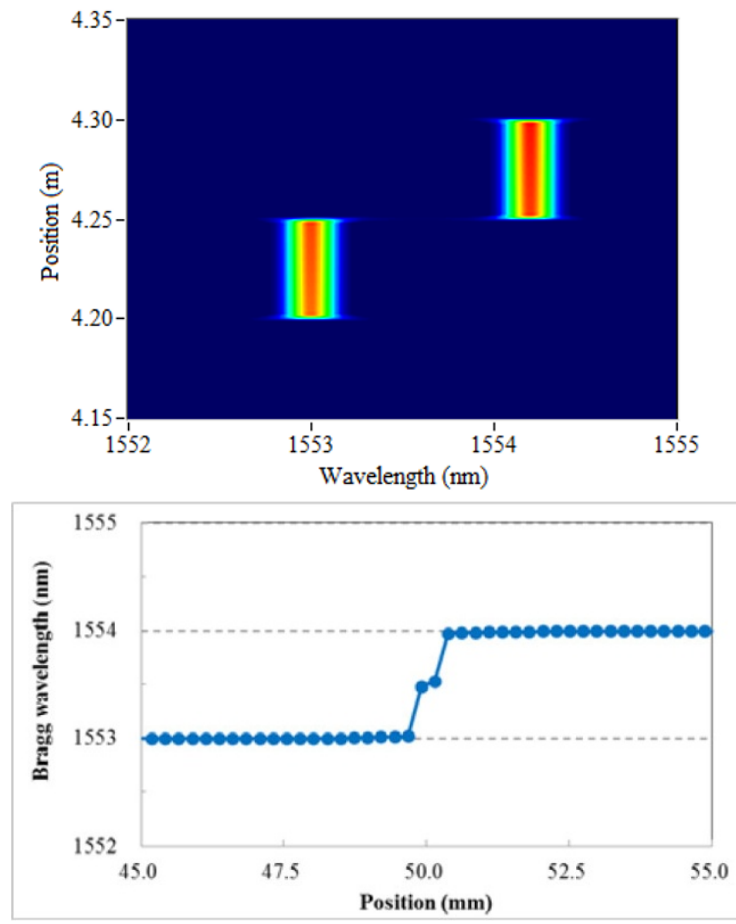

(c) Window length $=600 \mathrm{pm}$

Fig. 25 Spectrograms and Bragg wavelength distributions when the 1.0-nm Bragg wavelength shift was simulated at the center of the 100-mm FBG.

\subsection{Simultaneous strain and temperature measurement}

Generally, since fiber-optic distributed strain sensors have a cross-sensitivity toward strain and temperature, some techniques to compensate for temperature differences or to discriminate strain and temperature become necessary to perform accurate and stable measurement at practical fields.

A number of strain and temperature discrimination techniques using a single fiber have been proposed. Most of them use two kinds of FBGs in a single fiber, such as two FBGs in claddings with different diameters [44], two FBGs in parts of a fiber with different dopants [45], two FBGs with one specially packaged [46, 47], and two FBGs of different refractive index profiles [48, 49]. In addition, there are techniques using a single FBG. Echevarria et al. used the first and second order diffraction wavelength of an FBG [50], Chehura et al. used a tilted FBG and measured spectra of 
core-core mode coupling and core-cladding mode coupling [51], and Sudo et al. inscribed an FBG into a polarization-maintaining (PM) fiber and measured Bragg wavelengths of two different polarization modes [52]. However, these techniques have not been investigated thoroughly for the use of distributed sensing.

When FBGs are inscribed into a PANDA fiber (PANDA-FBG), they reflect two Bragg wavelengths corresponding to two polarization modes, fast and slow modes, due to the birefringence effect in the PANDA fiber. Two measurands of strain and temperature are determined by observing two Bragg wavelengths, which have different sensitivities towards strain and temperature between each mode [52]. In principle, this technique is compatible with the distributed sensing technique based on the OFDR. When we introduce PANDA-FBG into the sensing system, besides using PANDA fibers instead of single mode fibers and detecting two Bragg wavelengths instead of one, it does not require any system modifications. One of the most advantageous factors of this changeover is that the high spatial is sustained.

In this paper, the application of the simultaneous strain and temperature measurement technique using a PANDA-FBG to distributed sensing based on the OFDR [20] is shown.

\subsubsection{Principle of simultaneous strain and temperature measurement using PANDA-FBG}

A PANDA fiber has two polarization modes, which are called as fast and slow modes, due to the birefringence effect in the fiber. Since refractive indices of fast and slow modes are different, an FBG inscribed into a PANDA fiber has two distinct Bragg wavelengths corresponding to each mode. Each Bragg wavelength also has different sensitivities to strain and temperature. Therefore, strain and temperature can be determined at the same time by observing the two Bragg wavelengths.

The shift in the fast mode Bragg wavelength, $\Delta \lambda_{f}$, and that of the slow mode Bragg wavelength, $\Delta \lambda_{s}$, induced by strain and temperature changes, $\Delta \varepsilon$ and $\Delta T$, respectively, are expressed as

$$
\left(\begin{array}{l}
\Delta \lambda_{f} \\
\Delta \lambda_{s}
\end{array}\right)=\left(\begin{array}{ll}
K_{\varepsilon f} & K_{T f} \\
K_{\varepsilon s} & K_{T s}
\end{array}\right)\left(\begin{array}{c}
\Delta \varepsilon \\
\Delta T
\end{array}\right)=\mathbf{K}\left(\begin{array}{c}
\Delta \varepsilon \\
\Delta T
\end{array}\right)
$$

where $K$ represents the sensitivity. The first subscript of $K$ indicates strain or temperature, and the second one indicates each mode. Once the $\mathbf{K}$ matrix is determined, a pair of observed Bragg wavelengths can determine strain and temperature by taking the inverse matrix of $\mathbf{K}$ and calculating

$$
\left(\begin{array}{c}
\Delta \varepsilon \\
\Delta T
\end{array}\right)=\mathbf{K}^{-1}\left(\begin{array}{c}
\Delta \lambda_{f} \\
\Delta \lambda_{s}
\end{array}\right)
$$

\subsubsection{Experimental setup and results}

In order to investigate the simultaneous strain and temperature distributed measurements using the PANDA-FBG, it was bonded to a tensile specimen, and the measurement was implemented during the tensile test as changing temperature.

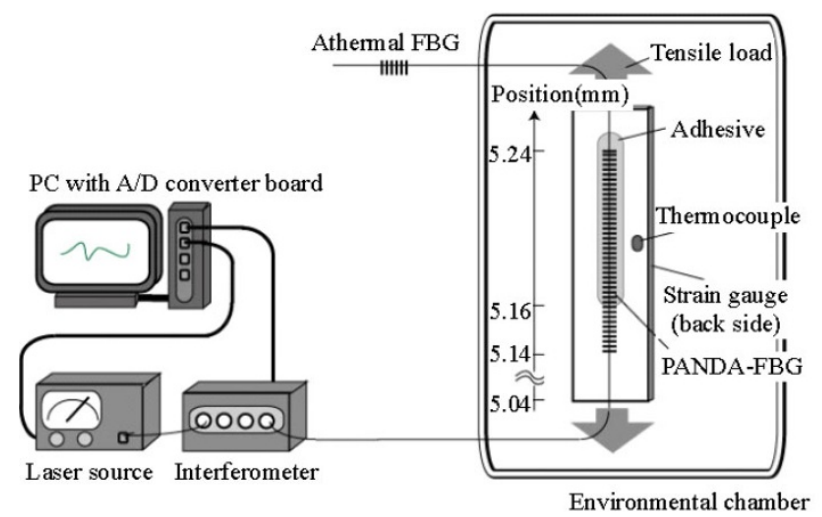

Fig. 26 Schematic of the experimental setup for simultaneous strain and temperature measurement

The experimental setup is shown in Fig. 26. The position represents the distance from $\mathrm{R} 3$ as seen in Fig. 7. An additional FBG, expressed as "athermal FBG", was prepared. The athermal FBG is a special package, which consists of an assembly of materials with different thermal expansion coefficients and reduces Bragg wavelength shifts against temperature changes. It was used to compensate wavelength drifts caused by the laser source. A 100-mm-length 
PANDA-FBG was bonded to a stainless tensile plate with a polyester adhesive. The length, width, and thickness of the plate were $330 \mathrm{~mm}, 40 \mathrm{~mm}$, and $5 \mathrm{~mm}$, respectively. The location of the PANDA-FBG was from $5.14 \mathrm{~m}$ to $5.24 \mathrm{~m}$ position $\left(5.14 \mathrm{~m}<L_{i}<5.24 \mathrm{~m}\right)$. A portion of the PANDA-FBG sensor (from $5.14 \mathrm{~m}$ to $5.16 \mathrm{~m}$ position) was not bonded on the plate to be in the stress-free condition.

The environmental chamber was fixed to the universal testing machine. The inside dimension of the environmental chamber was $355 \mathrm{~W} \times 430 \mathrm{D}$ $\times 550 \mathrm{H}(\mathrm{mm})$. A few hours of dwell time was taken for each temperature condition. Temperature was being monitored by a temperature sensor of the chamber and a thermocouple attached on the steel plate. Two thermocouples were attached at the point where $L_{i}$ is $5.16 \mathrm{~m}$ and $5.24 \mathrm{~m}$. When temperature variations of $50^{\circ} \mathrm{C}, 75^{\circ} \mathrm{C}$, and $100{ }^{\circ} \mathrm{C}$ were applied, the thermocouple at $L_{i}=5.24 \mathrm{~m}$ showed higher temperature than the other for $1.2^{\circ} \mathrm{C}, 1.7^{\circ} \mathrm{C}$, and $2.6^{\circ} \mathrm{C}$, respectively.

The tensile plate to which the PANDA-FBG was bonded was set into an environmental chamber, and several temperature variations were applied to it. Several variations of static tensile loads were applied to the plate at each temperature condition. Applied temperature variations were room temperature $\left(23{ }^{\circ} \mathrm{C}\right), 50{ }^{\circ} \mathrm{C}, 75^{\circ} \mathrm{C}$, and $100{ }^{\circ} \mathrm{C}$, and tensile load variations were $0 \mathrm{kN}, 8 \mathrm{kN}, 16 \mathrm{kN}, 24 \mathrm{kN}$, and $32 \mathrm{kN}$, thus experiments under 20 cases in total were implemented.

The components of $\mathbf{K}^{-1}$ were determined by using measurement data of 20 cases, and it is given by

$$
\mathbf{K}^{-1}=\left(\begin{array}{cc}
-8098 & 8860 \\
10768 & -10635
\end{array}\right)
$$

where the unit of components is $\mu \varepsilon / \mathrm{nm}$.

The averaged strain and temperature over the bonded part of the FBG length were determined by (21), and the results are shown in Fig. 27. The vertical axes represent measured values, and the horizontal axes show conditions; thus, the solid lines indicate the ideal values without errors. The errors in the strain measurements were from $-100 \mu \varepsilon$ to $69 \mu \varepsilon$, and those in temperature measurements were from $-5.7^{\circ} \mathrm{C}$ to $6.1{ }^{\circ} \mathrm{C}$
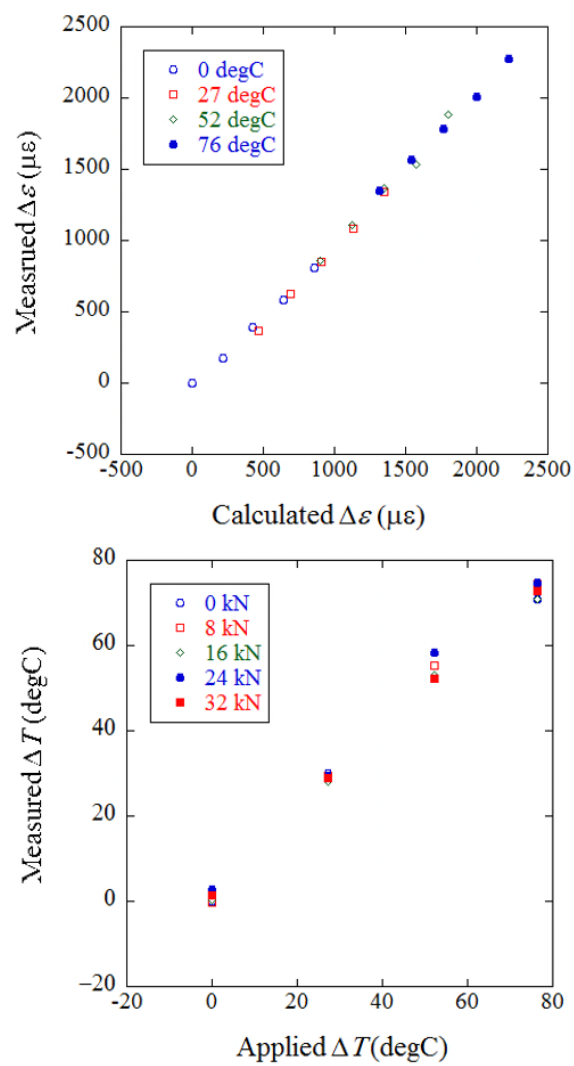

Fig. 27 Simultaneous measurement results (top: strain, bottom: temperature).

Strain and temperature distributions along the FBG bonded were determined as well. Figure 28 shows the results of the simultaneous distributed measurement of a test case. The applied strain, $\Delta \varepsilon$, was $1997 \mu \varepsilon$, and the temperature difference from the room temperature, $\Delta T$, was $76.3{ }^{\circ} \mathrm{C}$. They are represented as the solid line in the figure. Measured values were obtained by the interval of $0.76 \mathrm{~mm}$ and correlated with conditions. This result demonstrates the applicability of simultaneous strain and temperature distributed sensing. Variations of the measured value along the position, however, were observed. In order to improve the measurement accuracy and enhance the applicability, the accuracy of observing the Bragg wavelengths and the 
condition number of $\mathbf{K}$ should be improved.
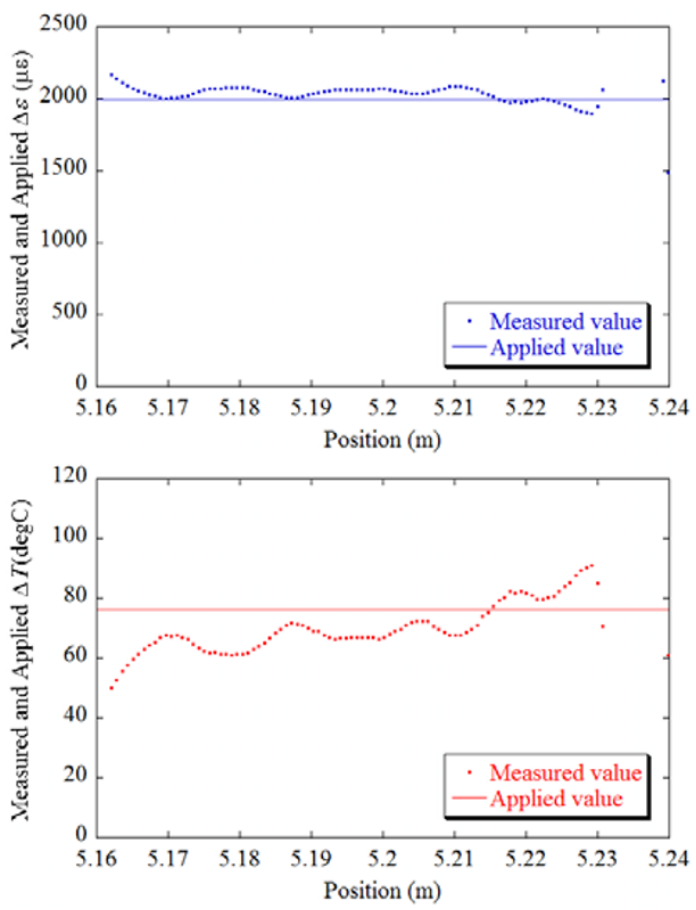

Fig. 28 Results of simultaneous distributed sensing under the case in which $1997 \mu \varepsilon$ and $76.3^{\circ} \mathrm{C}$ were applied (top: strain, bottom: temperature).

\section{Conclusions}

In this paper, we reviewed our researches on the topics of the SHM with the fiber-optic distributed strain sensor. Highly-dense information on strains in a structure is useful to identify some kind of existing damage or applied loads. In 1990s, however, the spatial resolution of the commercially available system could not be enough to detect damage based on strain distributions measured. The recent progress can make the spatial resolution of distributed sensors enough high to provide valuable information for damage detection and load identification.

The fiber-optic distributed sensors developed by the authors have been applied to damage detection of a single-lap joint and to load identification of a beam simply supported. Consequently, we confirmed that the applicability of the distributed sensor to the SHM could be improved as making the spatial resolution higher. In addition, we showed that the simulation techniques considering both structural and optical effects seamlessly in strain measurement can be powerful tools to evaluate the performance of a sensing system and design it for the SHM. Finally, the technique for simultaneous distributed strain and temperature measurement using the PANDA-FBG was shown, because problems caused by the cross-sensitivity toward strain and temperature would be always inevitable in strain measurement for the SHM. The authors think that to improve the accuracy in simultaneous measurements as keeping the high spatial resolution is still a problem to be addressed.

\section{Acknowledgment}

The Authors express their gratitude to Optics and Electronics Laboratory of Fujikura Ltd. for providing long-length FBGs.

Open Access This article is distributed under the terms of the Creative Commons Attribution License which permits any use, distribution, and reproduction in any medium, provided the original author(s) and source are credited.

\section{References}

[1] D. Balageas, C. P. Fritzen, and A. Gümes, Structural health monitoring. London, UK: ISTE, 2006, pp. 15-18.

[2] H. Murayama, G. Akiyama, H. Igawa, T. Nakamura, K. Kageyama, K. Uzawa, et al., "Application of inverse analysis of distributed load with strain sensors to wing structures," in Proc. of the 7th International Workshop on Structural Health Monitoring, vol. 1, pp. 75-82, 2009.

[3] C. R. Farrar and K. Worden, "An introduction to structural health monitoring," Philosophical Transactions of The Royal Society A, vol. 365, no. 1851, pp. 303-315, 2007.

[4] C. R. Farrar, S. W. Doebling, and D. A. Nix, "Vibration-based structural damage identification," Philosophical Transactions of The Royal Society A, vol. 359, no. 1778, pp. 131-149, 2001.

[5] J. Dakin and B. Culshaw, Optical fiber sensors, London, U. K: Artech House, vol. 4, 1997, pp. 309-407. 
[6] B. Glišić and D. Inaudi, Fiber optic methods for structural health monitoring. West Sussex, U. K.: John Wiley \& Sons, 2007, pp. 19-40.

[7] T. Horiguchi, T. Kurashima, and M. Tateda, "Tensile strain dependence of Brillouin frequency shift in silica optical fiber," IEEE Photonics Technology Letters, vol. 1, no. 5, pp. 107-108, 1989.

[8] M. LeBlanc, S. Huang, M. Ohn, and M. Measures, "Distributed strain measurement based on fiber Bragg grating and its reflection spectrum analysis," Optics Letters, vol. 21, no. 17, pp. 1405-1407, 1996.

[9] A. Nand, D. Kitcher, S. Wade, R. Jones, G. Baxter and S. Collins, "Localized measurements using an interrogation method to process intensity reflection spectra from chirped FBG," in Proc. SPIE, vol. 6619, pp. 661911-1-661911-4, 2007.

[10] S. Huang, M. Ohn, and M. Measures, "Phase-based Bragg intragrating distributed strain sensor," Applied Optics, vol. 35, no. 7, pp. 1135-1142, 1996.

[11] M. Volanthen, H. Geiger, and J. P. Dakin, "Distributed grating sensors using low-coherence reflectometry," Journal of Lightwave Technology, vol. 15, no. 11, pp. 2076-2082, 1997.

[12] R. M. Measures, M. M. Ohn, and S. Y. Huang, J. Bigue, and N. Y. Fan, "Tunable laser demodulation of various fiber Bragg grating sensing modalities," Smart Materials and Structures, vol. 7, no. 2, pp. 237-247, 1998.

[13] H. Murayama, K. Kageyama, H. Naruse, A. Shimada, and K. Uzawa, "Application of fiber-optic distributed sensors to health monitoring for full-scale composite structures," Journal of Intelligent Material Systems and Structures, vol. 14, no. 1, pp. 3-13, 2003.

[14] H. Murayama, K. Kageyama, H. Naruse, and A. Shimada, "Distributed strain sensing from damaged composite materials based on shape variation of the Brillouin spectrum," Journal of Intelligent Material Systems and Structures, vol. 15, no. 1, pp. 17-25, 2004.

[15] H. Igawa, H. Murayama, T. Kasai, I. Yamaguchi, K Kageyama, and K. Ohta, "Measurements of strain distributions with a long gauge FBG sensors using optical frequency domain reflectometry," in Proc. SPIE, vol. 5855, pp. 547-550, 2005.

[16] H. Murayama, K. Kageyama, K. Uzawa, K. Ohara, and H. Igawa, "Strain monitoring of a single-lap joint with embedded fiber-optic distributed sensors," Structural Health Monitoring, vol. 11, no. 3, pp. 322-341, 2012.
[17] H. Murayama, K. Tachibana, Y. Hirano, H. Igawa, K. Kageyama, K. Uzawa, et al., "Distributed strain and load monitoring of $6 \mathrm{~m}$ composite wing structures by FBG arrays and long-length FBGs," in Proc. SPIE, vol. 8421, pp. 84212D, 2012.

[18] D. Wada, H. Murayama, and H. Igawa, "Lateral load measurements based a distributed sensing system of optical frequency domain reflectometry using long-length fiber Bragg gratings," Journal of Lightwave Technology, vol. 30, no. 14, pp. 2337-2344, 2012.

[19] H. Igawa, K. Ohta, T. Kasai, I. Yamaguchi, H. Murayama, and K. Kageyama, "Distributed measurements with a long gauge FBG sensors using optical frequency domain reflectometry (1st report, system investigation using optical simulation model)," Journal of Solid Mechanics and Materials Engineering, vol. 2, no. 9, pp. 1242-1252, 2008.

[20] D. Wada, H. Murayama, H. Igawa, K. Kageyama, K. Uzawa, and K. Omichi, "Simultaneous distributed measurements of strain and temperature by polarization maintain fiber Bragg grating based on optical frequency domain reflectometry," Smart Materials and Structures, vol. 20, no. 8, pp. 085028-850358, 2011.

[21] H. Murayama, K. Kageyama, A. Shimada, and A. Nishiyama, "Improvement of spatial resolution for strain measurements by analyzing Brillouin gain spectrum," in Proc. SPIE, vol. 5855, pp. 551-554, 2005.

[22] Y. Sakairi, S. Matsuura, S. Adachi, and Y. Koyamada, "Prototype double-pulse BOTDR for measuring distributed strain with $20-\mathrm{cm}$ spatial resolution," in Proc. SICE Annual Conference, pp. 1106-1109, 2008.

[23] Y. Mizuno, Z. He, and K. Hotate, "Distributed strain measurement using a tellurite glass fiber with Brillouin optical correlation-domain analysis," Optics Communications, vol. 283, no. 11, pp. 2526-2528, 2010.

[24] S. Shen, Z. Wu, C. Yang, Y. Tang, G. Wu, and W. Hong, "A new optical fiber sensor with improved strain sensitivity based on distributed fiber sensing technique," in Proc. SPIE, vol. 7293, pp. 729315, 2009.

[25] Y. Dong, X. Bao, and W. Li, "Differential Brillouin gain for improvement the temperature accuracy and spatial resolution in a long-distance distributed fiber sensor," Applied Optics, vol. 48, no. 22, pp. 4297-4301, 2009.

[26] L. Li, J. Yang, Z. Zhang, X. Chen, and M. Zhang, 
"Kilometers-range dark-pulse Brillouin optical time domain analyzer with centimeters spatial resolution," in Proc. of 2010 Symposium on Photonics and Optoelectronics, Chengdu, China, June 19-21, pp. 1-4, 2010.

[27] K. Y. Song, Z. He, and K. Hotate, "Distributed strain measurement with millimeter-order spatial resolution based on Brillouin optical correlation domain analysis," Optics Letters, vol. 31, no. 17, pp. 2526-2528, 2010.

[28] B. A. Childers, M. E. Froggatt, S. G. Allison, T. C. Sr. Moore, D. A. Hare, C. F. Batten, et al., "Use of 3000 Bragg grating strain sensors distributed on four eight-meter optical fibers during static load tests of a composite structure," in Proc. SPIE, vol. 4332, pp. 133-142, 2001.

[29] M. Goland and E. Reissner, "The stresses in cemented joints," Journal of Applied Mechanics, vol. 11, no. 1, pp. A17-A27, 1944.

[30] L. J. Hart-Smith, "Adhesive bonded single-lap joints - technical report," NASA-CR-112236, 1973.

[31] W. A. Bigwood and A. D. Crocombe, "Non-linear adhesive bonded joint design analyses," International Journal of Adhesion and Adhesives, vol. 10, no. 1, pp. 31-41, 1990.

[32] H. Murayama, K. Ohara, N. Kanata, K. Kageyama, and H. Igawa, "Strain monitoring and defect detection in welded joints by using fiber-optic distributed sensors with high spatial resolution," E-Journal of Advanced Maintenance, vol. 2, no. 3, pp. 191-199, 2011.

[33] A. Tessler and J. L. Spangler, "Inverse FEM for full-field reconstruction of elastic deformation in shear deformable plates and shells," in Proc. of Second European Workshop on Structural Health Monitoring, Munich, Germany, July 7-9, pp. 83-90, 2004.

[34] C. W. Coates and P. Thamburaj, "Inverse method using finite strain measurements to detection flight load distribution functions," Journal of Aircraft, vol. 45, no. 2, pp. 366-370, 2008.

[35] T. Nakamura and H. Igawa, "Inverse analysis of distributed load using strain data," Journal of the Japan Society for Aeronautical and Space Sciences, vol. 56, no. 659, pp. 566-572, 2008 (in Japanese).

[36] H. Igawa, H. Murayama, T. Nakamura, I. Yamaguchi, K. Kageyama, K. Uzawa, D. Wada, et al., "Measurement of distributed strain and load identification using $1500 \mathrm{~mm}$ gauge length FBG and optical frequency domain reflectometry," in Proc. SPIE, vol. 7503, pp. 750351-1-750351-4,
2009.

[37] H. Murayama, K. Kageyama, S. Kobayashi, G. Akiyama, K. Ohara, I. Ohsawa, et al., "Application of distributed sensing technique with FBG sensors to structural health monitoring," in Proc. of the 6th International Workshop on Structural Health Monitoring, Stanford, USA, September 11-13, pp. 1020-1029, 2007.

[38] R. B. Wagreich, W. A. Atia, H. Singh, and J. S. Sirkis, "Effects of diametric load on fibre Bragg gratings fabricated in low birefringent fibre," Electronics Letters, vol. 32, no. 13, pp. 1223-1224, 1996.

[39] A. Zhang, B. Guan, X. Tao, and H. Tam, "Experimental and theoretical analysis of fiber Bragg gratings under lateral compression," Optics Communications, vol. 206, no. 1-3, pp. 81-87, 2002.

[40] R. Gafsi and M. A. El-Sherif, "Analysis of induced-birefringence effects on fiber Bragg gratings," Optical Fiber Technology, vol. 6, no. 3, pp. 299-323, 2000.

[41] D. Wada and H. Murayama, "Analytical investigation of response of birefringent fiber Bragg grating sensors in distributed monitoring system based on optical frequency domain reflectometry," Optics and Lasers in Engineering, in press, 2013.

[42] M. Yamada and K. Sakuda, "Analysis of almost-periodic distributed feedback slab waveguides via a fundamental matrix approach," Applied Optics, vol. 26, no. 16, pp. 3473-3478, 1987.

[43] T. Erdogan, "Fiber grating spectra," Journal of Lightwave Technology, vol. 15, no. 8, pp. 1277-1294, 1997.

[44] S. W. James, M. L. Dockney, and R. P. Tatam, "Simultaneous independent temperature and strain measurement using in-fiber Bragg grating sensors," Electronics Letters, vol. 32, no. 12, pp. 1133-1134, 1996.

[45] P. M. Cavaleiro, F. M. Araujo, L. A. Ferreira, J. L. Santos, and F. Farahi, "Simultaneous measurement of strain and temperature using Bragg gratings written in germanosilicate and boroncodoped germanosilicate fibers," IEEE Photonics Technology Letters, vol. 11, no. 12, pp. 1636-1637, 1999.

[46] M. Song, S. B. Lee, S. S. Choi, and B. Lee, "Simultaneous measurement of temperature and strain using two fiber Bragg gratings embedded in a glass tube," Optical Fiber Technology, vol. 3, no. 2, pp. 194-196, 1997. 
[47] B. O. Guan, H. Y. Tam, L. W. Chan, C. L. Choy, and M. S. Demokan, "Discrimination between strain and temperature with a single fiber Bragg grating," Microwave and Optical Technology Letters, vol. 33, no. 3, pp. 200-202, 2002.

[48] G. Simpson, K. Kalli, K. Zhou, L. Zhang, and I. Bennion, "Blank beam fabrication of regenerated type IA gratings," Measurement Science and Technology, vol. 15, no. 8, pp. 1665-1669, 2004.

[49] S. O. Park, B. W. Jang, Y. G. Lee, C. G. Kim, and C. Y. Park, "Simultaneous measurement of strain and temperature using a reverse index fiber Bragg grating sensor," Measurement Science and Technology, vol. 21, no. 3, pp. 035703, 2010.

[50] J. Echevarria, A. Quintela, C. Jauregui, and J. M.
Lopez-Higuera, "Uniform fiber Bragg grating firstand second-order diffraction wavelength experimental characterization for strain-temperature discrimination," IEEE Photonics Technology Letters, vol. 13, no. 7, pp. 696-698, 2001.

[51] E. Chehura, S. W. James, and R. P. Tatam, "Temperature and strain discrimination using a single tilted fibre Bragg grating," Optics Communication, vol. 275, no. 2, pp. 344-347, 2007.

[52] M. Sudo, M. Nakai, K. Himeno, S. Suzaki, A. Wada, and R. Yamauchi, "Simultaneous measurement of temperature and strain using PANDA fiber grating," in Proc. of the 12th International Conference on Optical Fiber Sensors, OSA Technical Digest, Virginia, USA, October 28, vol. 16, pp. 170-173, 1997. 